\title{
Jordan-Arens Irregularity
}

\author{
Chris Auger
}

A thesis submitted to the Faculty of Graduate Studies Carleton University in partial fulfillment of the requirements for the degree of Master of Science in the Ottawa-Carleton Institute for Graduate Studies and Research in Mathematics and Statistics

(C)2007 Chris Auger 


$\begin{array}{ll}\begin{array}{l}\text { Library and } \\ \text { Archives Canada }\end{array} & \begin{array}{l}\text { Bibliothèque et } \\ \text { Archives Canada }\end{array} \\ \begin{array}{l}\text { Published Heritage } \\ \text { Branch }\end{array} & \begin{array}{l}\text { Direction du } \\ \text { Patrimoine de l'édition }\end{array} \\ \begin{array}{l}\text { 395 Wellington Street } \\ \text { Ottawa ON K1A ON4 }\end{array} & \begin{array}{l}\text { 395, rue Wellington } \\ \text { Ottawa ON K1A ON4 } \\ \text { Canada }\end{array}\end{array}$

Your file Votre référence ISBN: 978-0-494-33690-8 Our file Notre référence ISBN: 978-0-494-33690-8

NOTICE:

The author has granted a nonexclusive license allowing Library and Archives Canada to reproduce, publish, archive, preserve, conserve, communicate to the public by telecommunication or on the Internet, loan, distribute and sell theses worldwide, for commercial or noncommercial purposes, in microform, paper, electronic and/or any other formats.

The author retains copyright ownership and moral rights in this thesis. Neither the thesis nor substantial extracts from it may be printed or otherwise reproduced without the author's permission.
AVIS:

L'auteur a accordé une licence non exclusive permettant à la Bibliothèque et Archives Canada de reproduire, publier, archiver, sauvegarder, conserver, transmettre au public par télécommunication ou par l'Internet, prêter, distribuer et vendre des thèses partout dans le monde, à des fins commerciales ou autres, sur support microforme, papier, électronique et/ou autres formats.

L'auteur conserve la propriété du droit d'auteur et des droits moraux qui protège cette thèse. $\mathrm{Ni}$ la thèse ni des extraits substantiels de celle-ci ne doivent être imprimés ou autrement reproduits sans son autorisation.
In compliance with the Canadian

Privacy Act some supporting forms may have been removed from this thesis.

While these forms may be included in the document page count, their removal does not represent any loss of content from the thesis.
Conformément à la loi canadienne sur la protection de la vie privée, quelques formulaires secondaires ont été enlevés de cette thèse.

Bien que ces formulaires aient inclus dans la pagination, il n'y aura aucun contenu manquant.

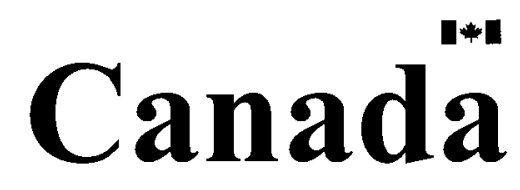




\section{Abstract}

The topological centres of the convolution algebra of nuclear operators has been studied recently primarily by Neufang. This thesis is primarily composed of three sections. The first section focuses on calculating the two topological centres of this algebra for discrete of groups. The second section uses this result to discuss the non-hereditary property of strong Arens irregularity to subalgebras. This is done by showing the existence of a strongly Arens irregular Banach algebra which admits a subalgebra that is not strongly Arens irregular. Finally, the last section introduces the notion of a Jordan topological centre of a Banach algebra and successfully shows that the convolution algebras of nuclear operators and $\ell_{1}(G)$ are both Jordan strongly Arens irregular. 


\section{Acknowledgements}

I would like to take this opportunity to thank my thesis supervisor Matthias Ne-

ufang. His guidance and patience throughout has been inspiring and was crucial to the completion of this thesis. 


\section{Contents}

1 Introduction 1

2 Preliminaries $\quad 3$

2.1 Banach algebras and modules . . . . . . . . . . . . 3

2.2 Arens products and topological centres . . . . . . . . . . 5

2.3 Nuclear operators . . . . . . . . . . . . . . . 10

3 The topological centres of the convolution algebra of nuclear operators $\quad 14$

3.1 The convolution product . . . . . . . . . . . . . 14

3.2 The first topological centre of $\mathcal{N}\left(L_{p}(G)\right) \ldots \ldots \ldots$

3.3 The second topological centre of $\mathcal{N}\left(L_{p}(G)\right) \ldots \ldots \ldots 21$

4 Strong Arens irregularity is not hereditary 31

4.1 Strongly Arens irregular Banach algebras with an Arens regular sub-

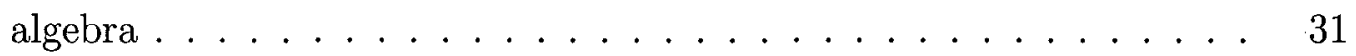

5 Jordan Topological Centres $\quad 38$

5.1 General results for Banach algebras . . . . . . . . . . . . 38

5.2 Jordan topological centre of $\ell_{1}(G) \ldots \ldots \ldots . \ldots . \ldots . \ldots 42$

5.3 Jordan topological centre of the convolution algebra of nuclear operators 52

5.4 Conclusion and main results ................. 53 


\section{Chapter 1}

\section{Introduction}

The Banach spaces $L_{p}(G)$, with $1 \leq p \leq \infty$ and $G$ a locally compact group, have been studied extensively as well as the Banach space $\mathcal{B}\left(L_{p}(G)\right)$, the space of bounded linear operators on $L_{p}(G)$. The projective tensor product $L_{p}(G) \otimes_{\gamma} L_{q}(G)$ (with $1<p, q<\infty$ being conjugate indices) can be identified with the space $\mathcal{N}\left(L_{p}(G)\right)$ of nuclear operators on $L_{p}(G)$ and, when $p=2$, with $\mathcal{H}=L_{2}(G)$ a Hilbert space, $\mathcal{N}\left(L_{2}(G)\right)$ coincides with $\mathcal{T}(\mathcal{H})$, the space of all trace class operators. In [18] M. Neufang introduces a new product on $\mathcal{N}\left(L_{p}(G)\right)$ and discusses many of the relations between $L_{1}(G)$ and $\mathcal{T}(\mathcal{H})$ suggesting that $\mathcal{T}(\mathcal{H})$ with this product is a noncommutative version of the group algebra $L_{1}(G)$. Thus we may view $\mathcal{T}\left(\ell_{2}(G)\right)$ with the usual composition as a noncommutative version of $\ell_{1}(G)$ with the pointwise product and $\mathcal{T}\left(L_{2}(G)\right)$ with this new product as a noncommutative version of $L_{1}(G)$ with the convolution product. It is with this view that we denote this new product by * (the usual symbol for convolution) and describe it as a convolution type product on $\mathcal{N}\left(L_{p}(G)\right)$. We would like to point out that this algebra has been studied subsequently by others including Aristov in [1] and Pirkovskii in [22] where in these papers they look at biprojectivity and biflatness for $\left(\mathcal{N}\left(L_{2}(G)\right), *\right)$. Their results are similar to those of $\left(L_{1}(G), *\right)$ which strengthens the analogy between the two convolution algebras.

In [18] Neufang also discusses briefly the relation of the topological centres of $L_{1}(G)$ and $\mathcal{N}\left(L_{p}(G)\right)$ with the different products. This relation is as follows: both 
$\left(\ell_{1}(G), \cdot\right)$, where $\cdot$ is the pointwise product, and $\left(\mathcal{N}\left(L_{p}(G), \circ\right)\right)$ are Arens regular; also for $|G|<\infty$ both $\left(L_{1}(G), *\right)$ and $\left(\mathcal{N}\left(L_{p}(G)\right), *\right)$ are (trivially) Arens regular. Now, the topological centre of $\left(L_{1}(G), *\right)$ has been studied primarily by Lau and Losert in [13] and more recently using a different approach by Neufang in [19] where it is shown that for any locally compact group $G,\left(L_{1}(G), *\right)$ is left and right strongly Arens irregular. In [15] Neufang shows that $\left(\mathcal{N}\left(L_{p}(G)\right)\right.$,*) is left strongly Arens irregular but not right strongly Arens irregular. In [5], Dales and Lau develop a construction of a Banach algebra that is left but not right strongly Arens irregular where they mention that the convolution algebra of nuclear operators developed by Neufang is an earlier example of an algebra having this property.

In this thesis we shall give the necessary definitions and some previous results about topological centres in section 2 that will be used throughout. In section 3 we will provide the details for showing that for a locally compact, noncompact group $G$, $\left(\mathcal{N}\left(L_{p}(G), *\right)\right)$ is left strongly Arens irregular but not right strongly Arens irregular as is done in [15]. In section 4 we will construct, by using a result by Dales and Lau [5], a Banach algebra that is strongly Arens irregular while admitting a subalgebra that is Arens regular and not strongly Arens irregular, which answers in the affirmative a question (not published) raised by Neufang. In section 5 we look at the Jordan algebra of a given Banach algebra. By forcing a Banach algebra to be commutative, via the Jordan product, we look at these effects in the bidual level. The motivation for this comes from the convolution algebra of the nuclear operators. As was stated above, in [19], Neufang shows that this algebra is left but not right strongly Arens irregular and we look at how this affects the continuity of the Jordan product extended to the bidual level. We introduce the notion of the Jordan topological centre of a Banach algebra and determine the Jordan topological centre of $\left(L_{1}(G), *\right)$ and $\left(\mathcal{N}\left(L_{p}(G)\right), *\right)$ for two different classes of groups. The technique that we use to determine the Jordan topological centre for these algebras is of interest on its own which is a strengthening of the factorization result developed in [18] by Neufang. 


\section{Chapter 2}

\section{Preliminaries}

In this section we give some of the definitions required throughout this thesis. Other definitions will be stated as needed. As well, we provide a few basic well known results that will be needed later. Although we provide these definitions and basic results, some background knowledge of these areas are assumed. For a more thorough introduction to Banach algebras we refer the reader to [4] and [21], and for measure theory to [3] and [11].

\subsection{Banach algebras and modules}

Definition 2.1.1 An algebra over $\mathbb{F}$ is a vector space $A$ over a field $\mathbb{F}$ such that there is a multiplication between elements that is distributive and associative, i.e. $\forall x, y, z \in A$, we have

$$
\begin{aligned}
& \text { i) } x(y+z)=x y+x z \\
& \text { ii) }(x+y) z=x z+y z \\
& \text { iii) } \quad(x y) z=x(y z)
\end{aligned}
$$

and is linked to scalar multiplication in an obvious way, i.e. $\forall \alpha \in \mathbb{F}$, we have

$$
(\alpha x) y=\alpha(x y)=x(\alpha y)
$$

Note: For this thesis, when the term algebra is used, it refers to a algebra over C. 
Definition 2.1.2 A Banach space is a complete normed space.

Definition 2.1.3 A Banach algebra is an algebra A, together with a norm \|\| , such that $(A,\|\|)$ is a Banach space and $\forall x, y \in A,\|x y\| \leq\|x\|\|y\|$.

Note: We can take as a definition of a Banach algebra to be a Banach space $A$, with norm \| \|, along with an algebra structure where $\exists K>0$ such that $\forall x, y \in A$, $\|x y\| \leq K\|x\|\|y\|$ since by putting ||| $\|\mid=K\| \|$ we get the desired inequality. There is no problem in doing this since scaling a norm does not change any of its topological properties.

Definition 2.1.4 Let $A$ be an algebra. A left $\boldsymbol{A}$-module is a vector space $X$ such that there is an action of $A$ on $X$ (i.e. a map $A \times X \rightarrow X$ ) which is denoted by $a \cdot x$ for all $a \in A$ and $x \in X$ which satisfies the following:

i) $(a+b) \cdot x=a \cdot x+b \cdot x, \quad \forall a, b \in A, \forall x \in X$,

ii) $(a b) \cdot x=a \cdot(b \cdot x), \quad \forall a, b \in A, \forall x \in X$,

iii) $a \cdot(x+y)=a \cdot x+a \cdot y, \quad \forall a \in A, \forall x, y \in X$,

and if $A$ has a unit, then

iv) $1 \cdot x=x, \quad \forall x \in X$

There is an analogous definition for a right A-module in which the multiplication of the elements between $A$ and $X$ are reversed, i.e. the action of $A$ on $X$ is denoted by $x \cdot a$ for all $a \in A$ and for all $x \in X$.

Definition 2.1.5 An A-bimodule $X$ is both a left A-module and a right A-module such that

$$
(a \cdot x) \cdot b=a \cdot(x \cdot b) \quad \forall a, b \in A \forall x \in X
$$

Definition 2.1.6 Let $A$ be a Banach space. A Banach A-bimodule $X$ is a Banach space $X$ such that $X$ is an $A$-bimodule, and the operation

$$
A \times X \times A \rightarrow X, \quad(a, x, b) \mapsto a \cdot x \cdot b
$$

is jointly continuous, where $A \times X \times A$ carries the product topology. 
Example: Let $A$ be a Banach algebra. Then

$$
A^{*}=\{f: A \rightarrow \mathbb{C} \mid f \text { is linear and continuous }\},
$$

the topological dual of $A$, is a Banach $A$-bimodule in the following sense: for $a, b \in A$ and $f \in A^{*}$

$$
\langle a \cdot f, b\rangle=\langle f, b a\rangle
$$

and

$$
\langle f \cdot a, b\rangle=\langle f, a b\rangle
$$

where $\langle$,$\rangle is the dual pairing of A^{*}$ and $A$ (i.e. $\langle f, a\rangle:=f(a)$ ).

One can define similar actions of $A^{* *}$ on $A^{*}$ making $A^{*}$ a Banach $A^{* *}$-bimodule and we explore this in the next section.

\subsection{Arens products and topological centres}

Let $(A, *)$ be a Banach algebra. The Arens products are defined on the bidual $A^{* *}$ of $A$ via module actions of $A^{* *}$ and $A$ on $A^{*}$.

Definition 2.2.1 The first (or left) Arens product, denoted by $\odot$, is defined as follows. Let $m, n \in A^{* *}, h \in A^{*}, f, g \in A$, then $m \odot n \in A^{* *}, n \odot h \in A^{*}$ and $h \odot f \in A^{*}$ are given by

$$
\begin{gathered}
\langle m \odot n, h\rangle:=\langle m, n \odot h\rangle \\
\langle n \odot h, f\rangle:=\langle n, h \odot f\rangle \\
\langle h \odot f, g\rangle:=\langle h, f * g\rangle .
\end{gathered}
$$

Definition 2.2.2 The second (or right) Arens product, denoted by $\diamond$, is defined as follows. Let $m, n \in A^{* *}, h \in A^{*}, f, g \in A$, then $m \diamond n \in A^{* *}, h \diamond m \in A^{*}$ and $f \diamond h \in A^{*}$ are given by

$$
\begin{aligned}
& \langle m \diamond n, h\rangle:=\langle n, h \diamond m\rangle \\
& \langle h \diamond m, f\rangle:=\langle m, f \diamond h\rangle \\
& \langle f \diamond h, g\rangle:=\langle h, g * f\rangle .
\end{aligned}
$$


In order to show that these two products on $A^{* *}$ are extensions of the original product on $A$, let $m, n \in A$ and $f \in A^{*}$. When we write $\langle m, f\rangle$ we mean $\langle\hat{m}, f\rangle$ where the map $m \mapsto \hat{m}$ is the canonical embedding of $A$ into $A^{* *}$. Then

$$
\begin{aligned}
\langle m \odot n, f\rangle & =\langle m, n \odot f\rangle \\
& =\langle n \odot f, m\rangle \\
& =\langle n, f \odot m\rangle \\
& =\langle f \odot m, n\rangle \\
& =\langle f, m * n\rangle \\
& =\langle m * n, f\rangle
\end{aligned}
$$

and

$$
\begin{aligned}
\langle m \diamond n, f\rangle & =\langle n, f \diamond m\rangle \\
& =\langle f \diamond m, n\rangle \\
& =\langle m, n \diamond f\rangle \\
& =\langle n \diamond f, m\rangle \\
& =\langle f, m * n\rangle \\
& =\langle m * n, f\rangle
\end{aligned}
$$

Definition 2.2.3 Let $X$ be a Banach space. We say that a net $\left(\phi_{\alpha}\right)_{\alpha \in I} \subset X^{*}$ converges $\mathbf{w}^{*}$ to some element $\phi$ if for every $f \in X$ we have that $\left\langle\phi_{\alpha}, f\right\rangle \longrightarrow\langle\phi, f\rangle$.

It is clear by the definitions of the Arens products that the maps $n \mapsto n \odot m$ and $n \mapsto m \diamond n$ aro $w^{*}-w^{*}$ continuous for evcry $m \in A^{* *}$, and so we define the first and second topological centres as follows.

Definition 2.2.4 The first topological centre of $A^{* *}$, denoted by $Z_{t}^{1}\left(A^{* *}\right)$, is the set

$$
Z_{t}^{1}\left(A^{* *}\right)=\left\{m \in A^{* *} \mid \text { the map } n \mapsto m \odot n \text { is } w^{*}-w^{*} \text { continuous }\right\}
$$


Definition 2.2.5 The second topological centre of $A^{* *}$, denoted by $Z_{t}^{2}\left(A^{* *}\right)$, is the set

$$
Z_{t}^{2}\left(A^{* *}\right)=\left\{m \in A^{* *} \mid \text { the map } n \mapsto n \diamond m \text { is } w^{*}-w^{*} \text { continuous }\right\} .
$$

Using the same techniques employed to show that the Arens products are extensions of the original product, we will now show that $A$ is always contained in each of the centres.

Proposition 2.2.1 Let $A$ be a Banach algebra. Then $A \subset Z_{t}^{1}\left(A^{* *}\right) \cap Z_{t}^{2}\left(A^{* *}\right)$.

Proof Let $m \in A$ and let $\left(n_{\alpha}\right)_{\alpha \in I}$ be a net in $A^{* *}$ converging $w^{*}$ to 0 . We have to show that $m \odot n_{\alpha} \longrightarrow 0\left(w^{*}\right)$. For every $f \in A^{*}$ we have

$$
\begin{aligned}
\left\langle m \odot n_{\alpha}, f\right\rangle & =\left\langle m, n_{\alpha} \odot f\right\rangle \\
& =\left\langle n_{\alpha} \odot f, m\right\rangle \\
& =\left\langle n_{\alpha}, f \odot m\right\rangle \longrightarrow 0 .
\end{aligned}
$$

Similarly,

$$
\begin{aligned}
\left\langle n_{\alpha} \diamond m, f\right\rangle & =\left\langle m, f \diamond n_{\alpha}\right\rangle \\
& =\left\langle f \diamond n_{\alpha}, m\right\rangle \\
& =\left\langle n_{\alpha}, m \diamond f\right\rangle \longrightarrow 0
\end{aligned}
$$

This shows that $m \in Z_{t}^{1}\left(A^{* *}\right) \cap Z_{t}^{2}\left(A^{* *}\right)$.

There is an alternative way of describing the first and second topological centres of $A^{* *}$, and we will show this next by using Theorem 8.6 in [2] which gives $\bar{A}^{w^{*}}=A^{* *}$ as Banach spaces.

Proposition 2.2.2 Let $A$ be a Banach algebra. Then

$$
\begin{aligned}
& \text { i) } Z_{t}^{1}\left(A^{* *}\right)=\left\{m \in A^{* *} \mid m \odot n=m \diamond n \quad \forall n \in A^{* *}\right\}, \text { and } \\
& \text { ii) } Z_{t}^{2}\left(A^{* *}\right)=\left\{m \in A^{* *} \mid n \odot m=n \diamond m \quad \forall n \in A^{* *}\right\} .
\end{aligned}
$$


Proof Let $m \in Z_{t}^{1}\left(A^{* *}\right)$ and $n \in A^{* *}$. Let $\left(m_{\alpha}\right)_{\alpha \in I}$ and $\left(n_{\beta}\right)_{\beta \in J}$ be nets in $A$ such that $m_{\alpha} \rightarrow m$ and $n_{\beta} \rightarrow n\left(w^{*}\right)$. Then

$$
\begin{array}{rlrl}
m \diamond n & =w^{*}-\lim _{\beta}\left(m \diamond n_{\beta}\right) & \\
& =w^{*}-\lim _{\beta}\left(w^{*}-\lim _{\alpha}\left(m_{\alpha} \diamond n_{\beta}\right)\right) & & \left(n_{\beta} \in A \subset Z_{t}^{2}\left(A^{* *}\right)\right) \\
& =w^{*}-\lim _{\beta}\left(w^{*}-\lim _{\alpha}\left(m_{\alpha} \odot n_{\beta}\right)\right) & & \left(m_{\alpha}, n_{\beta} \in A \quad \forall \alpha, \beta\right) \\
& =w^{*}-\lim _{\beta}\left(m \odot n_{\beta}\right) & & \\
& =m \odot n . & & \left(m \in Z_{t}^{1}\left(A^{* *}\right)\right)
\end{array}
$$

Similarly, assuming now that $m \in Z_{t}^{2}\left(A^{* *}\right)$,

$$
\begin{aligned}
n \diamond m & =w^{*}-\lim _{\beta}\left(n_{\beta} \diamond m\right) & & \left(m \in Z_{t}^{2}\left(A^{* *}\right)\right) \\
& =w^{*}-\lim _{\beta}\left(w^{*}-\lim _{\alpha}\left(n_{\beta} * m_{\alpha}\right)\right) & & \\
& =w^{*}-\lim _{\beta}\left(w^{*}-\lim _{\alpha}\left(n_{\beta} \odot m_{\alpha}\right)\right) & & \left(m_{\alpha}, n_{\beta} \in A \forall \alpha, \beta\right) \\
& =w^{*}-\lim _{\beta}\left(n_{\beta} \odot m\right) & & \left(n_{\beta} \in A \subset Z_{t}^{1}\left(A^{* *}\right)\right) \\
& =n \odot m & &
\end{aligned}
$$

The other directions are clear.

Corollary 1 Both $Z_{t}^{1}\left(A^{* *}\right)$ and $Z_{t}^{2}\left(A^{* *}\right)$ are subalgebras of $A^{* *}$ on which $\odot$ and $\diamond$ coincide.

Definition 2.2.6 Let $A$ be a Banach algebra. Then $A$ is called left (right) strongly Arens irregular, abbreviated $L S A I(R S A I)$, if $Z_{t}^{1}\left(A^{* *}\right)=A\left(Z_{t}^{2}\left(A^{* *}\right)=A\right)$. The algebra $A$ is called strongly Arens irregular if $Z_{t}^{1}\left(A^{* *}\right)=Z_{t}^{2}\left(A^{* *}\right)=A$ and called Arens regular if $Z_{t}^{1}\left(A^{* *}\right)=Z_{t}^{2}\left(A^{* *}\right)=A^{* *}$.

We will now prove that $L_{1}(G)$ is strongly Arens irregular, a result that will be used frequently in this thesis. This result has been shown by Lau and Losert in [13] and by Neufang in [18] and [19] and we give a general proof for any Banach algebra that has two fundamental properties which will now be defined (cf. [17]).

Definition 2.2.7 ([17]) Let $X$ be a Banach space and $\kappa$ a cardinal number.

i) A functional $f \in X^{* *}$ is called $w^{*}-\kappa$ continuous if $\forall$ nets $\left(x_{\alpha}\right)_{\alpha \in I} \subset \operatorname{Ball}\left(X^{*}\right)$ with 
$\aleph_{0} \leq|I| \leq \kappa$ such that $x_{\alpha} \longrightarrow 0\left(w^{*}\right)$, we have $\left\langle f, x_{\alpha}\right\rangle \longrightarrow 0$.

ii) We say that $X$ has Mazur's property of level $\kappa$ if every functional in $X^{* *}$ which is $w^{*}-\kappa$-continuous, actually belongs to $X$.

Definition 2.2.8 ([17]) Let $A$ be a Banach algebra and $\kappa$ a cardinal number. We say that $A^{*}$ has the left $A^{* *}$-factorization property of level $\kappa$ if

$$
\forall\left(h_{i}\right)_{i \in I} \subset \operatorname{Ball}\left(A^{*}\right), \text { with }|I|=\kappa, \exists\left(\psi_{i}\right)_{i \in I} \subset \operatorname{Ball}\left(A^{* *}\right) \exists h \in A^{*}
$$

such that $h_{i}=\psi_{i} \odot h$ for all $i \in I$.

Similarly we say that $A^{*}$ has the right $A^{* *}$-factorization property of level $\kappa$ if

$$
\forall\left(h_{i}\right)_{i \in I} \subset \operatorname{Ball}\left(A^{*}\right),|I|=\kappa, \exists\left(\psi_{i}\right)_{i \in I} \subset \operatorname{Ball}\left(A^{* *}\right) \exists h \in A^{*}
$$

such that $h_{i}=h \diamond \psi_{i}$ for all $i \in I$.

Theorem 2.2.1 ([17]) Let $A$ be a Banach algebra with the Mazur property of level $\kappa \geq \aleph_{0}$. If $A^{*}$ has the left $A^{* *}$-factorization property of level $\kappa$ (resp. right $A^{* *}$ factorization property of level $\kappa$ ) then $A$ is LSAI (resp. RSAI).

Proof Let $m \in Z_{t}^{1}\left(A^{* *}\right)$ and take a net $\left(h_{\alpha}\right)_{\alpha \in I} \subset \operatorname{Ball}\left(A^{*}\right)$ with $|I| \leq \kappa$ which converges $w^{*}$ to 0 . Since $A$ has the Mazur property of level $\kappa$, we only need to show that $\left\langle m, h_{\alpha}\right\rangle$ converges to 0 . It is sufficient to show that every convergent subnet $\left(\left\langle m, h_{\alpha}\right\rangle\right)_{\alpha \in I}$ converges to 0 . Let $\left(\left\langle m, h_{\alpha_{\beta}}\right\rangle\right)_{\beta}$ be a convergent subnet. As $A^{*}$ has the left $A^{* *}$-factorization property of level $\kappa$ we have that $h_{\alpha}=\psi_{\alpha} \odot h$ with $\left(\psi_{\alpha}\right)_{\alpha \in I} \subset \operatorname{Ball}\left(A^{* *}\right)$ and $h \in A^{*}$. By Alaoglu's Theorem, there exists a $w^{*}$-convergent subnet $\left(\psi_{\alpha_{\beta_{\gamma}}}\right)_{\gamma}$. Let $E=w^{*}-\lim _{\gamma} \psi_{\alpha_{\beta_{\gamma}}} \in \operatorname{Ball}\left(A^{* *}\right)$. Now, using the fact that $h_{\alpha}$ converges $w^{*}$ to 0 , we have for all $a \in A$

$\langle E \odot h, a\rangle=\langle E, h \odot a\rangle=\lim _{\gamma}\left\langle\psi_{\alpha_{\beta_{\gamma}}}, h \odot a\right\rangle=\lim _{\gamma}\left\langle\psi_{\alpha_{\beta_{\gamma}}} \odot h, a\right\rangle=\lim _{\gamma}\left\langle h_{\alpha_{\beta_{\gamma}}}, a\right\rangle=0$. 
Thus $E \odot h=0$. So, as $m \in Z_{t}^{1}\left(A^{* *}\right)$ :

$$
\begin{aligned}
\lim _{\beta}\left\langle m, h_{\alpha_{\beta}}\right\rangle & =\lim _{\gamma}\left\langle m, h_{\alpha_{\beta_{\gamma}}}\right\rangle \\
& =\lim _{\gamma}\left\langle m, \psi_{\alpha_{\beta_{\gamma}}} \odot h\right\rangle \\
& =\lim _{\gamma}\left\langle m \odot \psi_{\alpha_{\beta_{\gamma}}}, h\right\rangle \\
& =\langle m \odot E, h\rangle \\
& =\langle m, E \odot h\rangle \\
& =0 .
\end{aligned}
$$

Hence, $m \in A$, and $A$ is LSAI.

The same arguement works when taking $m \in Z_{t}^{2}\left(A^{* *}\right)$ and using the factorization $h_{\alpha}=g \diamond \psi_{\alpha}$ with $g \in A^{*}$.

Corollary 2 ([17]) Let $G$ be a locally compact, non-compact group. Then $L_{1}(G)$ is $S A I$.

Proof By (Lemma 2.1, [19]) we know that $L_{1}(G)$ has the Mazur property of level $\kappa(G)$, where $\kappa(G)$ is the compact covering number of $G$. By [20] (Theorem 2.1) we have that $L_{1}(G)$ has the left $A^{* *}$-factorization property of level $\kappa(G)$ and minor changes to the proof (replacing right translations with left and an obvious alteration to the construction of the net) we get that $L_{1}(G)$ has the right $A^{* *}$-factorization property of level $\kappa(G)$. By the above theorem we have that $L_{1}(G)$ is both LSAI and RSAI and thus SAI.

\section{$2.3 \quad$ Nuclear operators}

We first start with some measure theory.

Definition 2.3.1 Let $(X, \mathscr{A}, \mu)$ be a measure space. A subset $A$ of $X$ is called $\mu$ null if there is an element $B \in \mathscr{A}$ such that $A \subset B$ and $\mu(B)=0$. We say that a subset $\mathcal{N} \subset X$ is locally $\mu$-null if for each $A \in \mathscr{A}$ with $\mu(A)<\infty$ the set $A \cap \mathcal{N}$ is $\mu-n u l l$. 
Definition 2.3.2 Let $X$ be a space, $\mathscr{A}$ be a $\sigma$-algebra of subsets of $X$, and $\mu$ be a measure defined on $\mathscr{A}$. A property of points in $X$ is said to hold $\mu$-almost everywhere (abbreviated $\mu$-a.e.) if the set of points for which the property does not hold is $\mu$-null.

The next theorem contains the definition of a left Haar measure as well as the existence of this measure for a locally compact group.

Theorem 2.3.1 ([3]) Let $G$ be a locally compact group and $\mathscr{A}$ a $\sigma$-algebra of subsets of $G$. Then there exists a left translation invariant regular Borel measure $\mu$ on $\mathscr{A}$ which is called a left Haar measure (i.e. $\mu(g A)=\mu(A) \forall A \in \mathscr{A}, \forall g \in G$ where $g A=\{g a \mid a \in A\})$.

Definition 2.3.3 Let $G$ be a locally compact group, let $\mu$ be a left Haar measure on $G$ and let $1 \leq p<\infty$. Then

$$
L_{p}(G)=\left\{f: G \rightarrow \mathbb{C} \mid f \text { is Borel measurable and } \int_{G}|f|^{p} d \mu<\infty\right\}
$$

and the norm \|\|$_{p}$ on $L_{p}(G)$ is given by $\|f\|_{p}^{p}=\int_{G}|f|^{p} d \mu$. For $p=\infty$ we define \|\|$_{\infty}$ by letting $\|f\|_{\infty}=\inf _{M \geq 0}\{\{g \in G|| f(g) \mid>M\}$ is locally $\mu-n u l l\}$, then

$$
L_{\infty}(G)=\left\{f: G \rightarrow \mathbb{C} \mid f \text { is Borel measurable and }\|f\|_{\infty}<\infty\right\}
$$

Note: The functions $f$ in the above definition are actually equivalence classes of functions $[f]$, where

$$
[f]=\{h: G \rightarrow \mathbb{C} \mid h \text { is Borel measurable and } f=h \quad \mu-\text { a.e. }\} .
$$

Also, with these definitions, we get the following well known results:

$$
L_{1}(G)^{*}=L_{\infty}(G)
$$

and for $1<p, q<\infty$ with $\frac{1}{p}+\frac{1}{q}=1$ (this is also referred to as $p$ and $q$ being conjugated):

$$
L_{p}(G)^{*}=L_{q}(G)
$$


Definition 2.3.4 Let $X$ and $Y$ be Banach spaces and let $Y \subseteq X^{*}$, then we denote the pre-annihilator as $Y_{\perp}$ and the annihilator as $Y^{\perp}$, where

$$
Y_{\perp}=\{x \in X \mid\langle y, x\rangle=0 \quad \forall y \in Y\}
$$

and

$$
Y^{\perp}=\left\{x \in X^{* *} \mid\langle x, y\rangle=0 \quad \forall y \in Y\right\}
$$

Definition 2.3.5 Let $X$ and $Y$ be Banach spaces. Then $\mathcal{B}(X, Y)$ denotes the Banach space of bounded linear operators from $X$ to $Y$. If $X=Y$ then we write

$$
\mathcal{B}(X, Y)=\mathcal{B}(X, X)=: \mathcal{B}(X)
$$

The nuclear operators on $L_{p}(G)$, denoted by $\mathcal{N}\left(L_{p}(G)\right.$ ), have a number of equivalent definitions. The one that we will take is given in the following.

Definition 2.3.6 $\mathcal{N}\left(L_{p}(G)\right)=L_{p}(G) \otimes_{\gamma} L_{q}(G)$ where $q \in(1, \infty)$ such that $\frac{1}{p}+\frac{1}{q}=1$ and $\otimes_{\gamma}$ is the projective tensor product.

The projective tensor product norm is defined as follows: For $x \in L_{p}(G) \otimes L_{q}(G)$,

$$
\|x\|_{\mathcal{N}}=\inf _{k \geq 0}\left\{\sum_{i=0}^{k}\left\|f_{i}\right\|_{p}\left\|g_{i}\right\|_{q} \mid x=\sum_{i=0}^{k} f_{i} \otimes g_{i}\right\} .
$$

So, the space of nuclear operators is then the completion of $L_{p}(G) \otimes L_{q}(G)$ with respect to this norm. There is a subtle point one should be aware of when looking at $L_{p}(G) \otimes_{\gamma} L_{q}(G)$ which is that an arbitrary element of $L_{p}(G) \otimes_{\gamma} L_{q}(G)$ has the form

$$
\lim _{k \rightarrow \infty} \sum_{i=0}^{n_{k}} f_{i}^{(k)} \otimes g_{i}^{(k)} \quad \text { (in norm topology). }
$$

Fortunately, because we are using the projective tensor product, by Proposition 2.8 in [24] this element actually has the form

$$
\lim _{k \rightarrow \infty} \sum_{i=0}^{k} f_{i} \otimes g_{i}
$$

Note: The way in which the nuclear operators evaluate an element in $L_{p}(G)$ is by the following: let $x \otimes y$ be an elementary tensor in $\mathcal{N}\left(L_{p}(G)\right)$ and take an element 
$f \in L_{p}(G)$. Then $x \otimes y(f)=\langle y, f\rangle x$, where $\langle y, f\rangle$ is the usual pairing of elements in $L_{q}(G) \cong L_{p}(G)^{*}$ and $L_{p}(G)$. Since $L_{p}(G) \otimes_{\gamma} L_{q}(G)$ is the completion of the set of all linear combinations of elementary tensors, this evaluation extends linearly to all elements of $L_{p}(G) \otimes_{\gamma} L_{q}(G)=\mathcal{N}\left(L_{p}(G)\right)$. Also, as Banach spaces, it is shown in ([24]) that $\mathcal{N}\left(L_{p}(G)\right)^{*}=B\left(L_{p}(G)\right)$. The dual pairing between $\mathcal{N}\left(L_{p}(G)\right)$ and $\mathcal{B}\left(L_{p}(G)\right)$ is given by the following. For $T \in \mathcal{B}\left(L_{p}(G)\right)$ and an elementary tensor $\rho=\xi \otimes \eta$ in $\mathcal{N}\left(L_{p}(G)\right)$ we have

$$
\langle T, \rho\rangle=\langle T, \xi \otimes \eta\rangle=\langle T \xi, \eta\rangle
$$

where the last dual pairing is the usual of $L_{p}(G)$ and $L_{q}(G)$. As the dual brackets are bilinear this extends linearly and by the continuity of $T$, it also extends to the completion.

Remark: When $p=2$, the space $\mathcal{N}\left(L_{2}(G)\right)$ is also referred to as the trace class operators $\mathcal{T}\left(L_{2}(G)\right)$ and is commonly written as $\mathcal{T}(\mathcal{H})$.

The spaces $L U C(G)$, of left uniformly continuous functions on $G, R U C(G)$, of right uniformly continuous functions on $G$ and $C_{0}(G)$, of continuous functions on $G$ vanishing at infinity will be used throughout this thesis and are defined as subspaces of $C_{b}(G)$, the bounded continuous functions on $G$ as follows.

\section{Definition 2.3.7}

$$
\operatorname{LUC}(G)=\left\{f \in C_{b}(G) \mid \text { the map } G \longrightarrow\left(C_{b}(G),\|\|_{\infty}\right), x \mapsto l_{x} f \text { is continuous }\right\}
$$

where $l_{x}: L_{\infty}(G) \rightarrow L_{\infty}(G)$ defined pointwise by $l_{x} f(y)=f(x y)$.

\section{Definition 2.3.8}

$$
\begin{aligned}
& \qquad R U C(G)=\left\{f \in C_{b}(G) \mid \text { the map } G \longrightarrow\left(C_{b}(G),\|\|_{\infty}\right), x \mapsto r_{x} f \text { is continuous }\right\} \\
& \text { where } r_{x}: L_{\infty}(G) \rightarrow L_{\infty}(G) \text { defined pointwise by } r_{x} f(y)=f(y x)
\end{aligned}
$$

\section{Definition 2.3.9}

$$
C_{0}(G)=\left\{f \in C_{b}(G) \mid \forall \varepsilon>0 \exists K \subset G \text { compact such that } \forall x \notin K:|f(x)|<\varepsilon\right\} \text {. }
$$




\section{Chapter 3}

\section{The topological centres of the convolution algebra of nuclear \\ operators}

\subsection{The convolution product}

In this section $G$ denotes an arbitrary locally compact group. We start by defining a product $*$ on the nuclear operators as follows. For $\rho, \tau \in \mathcal{N}\left(L_{p}(G)\right)$ and $T \in \mathcal{B}\left(L_{p}(G)\right)=\mathcal{N}\left(L_{p}(G)\right)^{*}$, we set

$$
\langle\rho * \tau, T\rangle:=\langle\tau, T \odot \rho\rangle
$$

where the module operation $\odot$ is defined to be

$$
T \odot \rho:=M_{\left\langle L_{t} T L_{t^{-1}}, \rho\right\rangle}
$$

with $M_{\left\langle L_{t} T L_{t^{-1}}, \rho\right\rangle}$ being the multiplication operator of the function $t \mapsto\left\langle L_{t} T L_{t^{-1}}, \rho\right\rangle$ and $L_{t}$ is used instead of $l_{t}$ when $T$ is an operator rather than a function. For example, if $f \in L_{p}(G)$ then

$$
\left(M_{\left\langle L_{t} T L_{t^{-1}}, \rho\right\rangle} f\right)(x)=\left\langle L_{x} T L_{x^{-1}}, \rho\right\rangle f(x)
$$

The next proposition explores a bit of the nature of the function $\left\langle L_{t} T L_{t^{-1}}, \rho\right\rangle$. 
Proposition 3.1.1 Let $\rho \in \mathcal{N}\left(L_{p}(G)\right)$ and $T \in \mathcal{B}\left(L_{p}(G)\right)$. Then $\gamma_{l}^{T, \rho} \in R U C(G)$ where $\gamma_{l}^{T, \rho}(t):=\left\langle L_{t} T L_{t^{-1}}, \rho\right\rangle$.

Proof Define $\gamma_{l}^{T, \rho}: G \rightarrow \mathbb{C}$ by $\gamma_{l}^{T, \rho}(t):=\left\langle L_{t} T L_{t^{-1}}, \rho\right\rangle$. Then

$$
\begin{aligned}
\left\|\gamma_{l}^{T, \rho}\right\|_{\infty} & =\sup _{t \in G}\left|\gamma_{l}^{T, \rho}(t)\right| \\
& =\sup _{t \in G}\left|\left\langle L_{t} T L_{t^{-1}}, \rho\right\rangle\right| \\
& \leq \sup _{t \in G}\left\|L_{t} T L_{t^{-1}}\right\|\|\rho\| \\
& \leq \sup _{t \in G}\left\|L_{t}\right\|\|T\|\left\|L_{t^{-1}}\right\|\|\rho\| \\
& =\|T\|\|\rho\| .
\end{aligned}
$$

So we have that $\gamma_{l}^{T, \rho} \in L_{\infty}(G)$. To show that in fact $\gamma_{l}^{T, \rho}$ is actually in $R U C(G)$ we let $\varepsilon>0$ be given. Recall that $\mathcal{N}\left(L_{p}(G)\right)=L_{p}(G) \otimes_{\gamma} L_{q}(G)$ where $\frac{1}{p}+\frac{1}{q}=1$ and for $\rho \in \mathcal{N}\left(L_{p}(G)\right)$ then $\rho=\lim _{k} \sum_{n=1}^{k} \xi_{n} \otimes \eta_{n}$ for some $\xi_{n} \in L_{p}(G)$ and $\eta_{n} \in L_{q}(G)$. Let $\rho_{k}=\sum_{n=1}^{k} \xi_{n} \otimes \eta_{n}$ then $\left\|\rho-\rho_{k}\right\|_{\mathcal{N}} \longrightarrow 0$ as $k \longrightarrow 0$. So, it suffices to check that $\left\langle L_{t} T L_{t^{-1}}, \rho\right\rangle \in R U C(G)$ for an elementary tensor since

$$
\left|\left\langle L_{t} T L_{t^{-1}}, \rho\right\rangle-\left\langle L_{t} T L_{t^{-1}}, \rho_{k}\right\rangle\right| \leq\|T\|\left\|\rho-\rho_{k}\right\|_{\mathcal{N}} .
$$

Now, because of the strong continuity of left translation on $L_{p}(G)$ [8] (Proposition 2.41), we know that there is a neighbourhood $U$ of $e$ such that for each $x \in U$ we have $\left\|L_{x} \xi-\xi\right\|_{p}<\frac{\varepsilon}{2\|T\|\|\eta\|_{q}}$ and $\left\|L_{x} \eta-\eta\right\|_{q}<\frac{\varepsilon}{2\|T\|\|\xi\|_{p}}$ where we assume that $\|T\|,\|\xi\|_{p}$, and $\|\eta\|_{q} \neq 0$. Since $G$ is a topological group, we know that there exists a symmetric neighbourhood $V \subset U$. Thus for each $x \in V \subset U$ (and thus $x^{-1} \in V \subset U$ ) we have,

$$
\begin{aligned}
& \left\|r_{x} \gamma_{l}^{T, \rho}-\gamma_{l}^{T, \rho}\right\|_{\infty} \\
= & \sup _{t \in G}\left|r_{x} \gamma_{l}^{T, \rho}(t)-\gamma_{l}^{T, \rho}(t)\right| \\
= & \sup _{t \in G}\left|\gamma_{l}^{T, \rho}(t x)-\gamma_{l}^{T, \rho}(t)\right| \\
= & \sup _{t \in G}\left|\left\langle L_{t x} T L_{(t x)^{-1}}, \rho\right\rangle-\left\langle L_{t} T L_{t^{-1}}, \rho\right\rangle\right| \\
= & \sup _{t \in G}\left|\left\langle L_{t x} T L_{(t x)^{-1}}, \xi \otimes \eta\right\rangle-\left\langle L_{t} T L_{t^{-1}}, \xi \otimes \eta\right\rangle\right|
\end{aligned}
$$




$$
\begin{aligned}
& =\sup _{t \in G}\left|\left\langle L_{t x} T L_{(t x)^{-1}} \xi, \eta\right\rangle-\left\langle L_{t} T L_{t^{-1}} \xi, \eta\right\rangle\right| \\
& =\sup _{t \in G}\left|\left\langle T L_{(t x)^{-1}} \xi, L_{(t x)^{-1}} \eta\right\rangle-\left\langle T L_{t^{-1}} \xi, L_{t^{-1}} \eta\right\rangle\right| \\
& =\sup _{t \in G} \mid\left\langle T L_{(t x)^{-1}} \xi, L_{(t x)^{-1}} \eta\right\rangle+\left\langle T L_{t^{-1}} \xi, L_{(t x)^{-1}} \eta\right\rangle-\left\langle T L_{t^{-1}} \xi, L_{(t x)^{-1}} \eta\right\rangle- \\
& \left.\leq \sup _{t \in G}\left\|T L_{(t x)^{-1}} \xi-T T L_{t^{-1}} \xi\right\|_{p}\left\|L_{(t x)^{-1}} \eta\right\|_{q}+\sup _{t \in G} \| L_{(t x)^{-1}} \eta-L_{t^{-1}} \eta\right\rangle \mid \\
& \leq \sup _{t \in G}\left\|L_{(t x)^{-1}} \xi-L_{t^{-1}} \xi\right\|_{q}\|T\|_{p}\|T\| \eta L_{t^{-1}} \xi\left\|_{p}+\sup _{t \in G}\right\| L_{(t x)^{-1}} \eta-L_{t^{-1}} \eta\left\|_{q}\right\| T\|\| \xi \|_{p} \\
& =\sup _{t \in G}\left\|L_{t^{-1}}\left(L_{x^{-1}} \xi\right)-L_{t^{-1}} \xi\right\|_{p}\|T\|\|\eta\|_{q}+\sup _{t \in G}\left\|L_{t^{-1}}\left(L_{x^{-1}} \eta\right)-L_{t^{-1}} \eta\right\|_{q}\|T\|\|\xi\|_{p} \\
& \leq\left\|L_{x^{-1}} \xi-\xi\right\|_{p}\|T\|\|\eta\|_{q}+\left\|L_{x^{-1}} \eta-\eta\right\|_{q}\|T\|\|\xi\|_{p} \\
& <\varepsilon .
\end{aligned}
$$

Since $\left\langle L_{t} T L_{t^{-1}}, \rho\right\rangle \in R U C(G)$ then we have that $T \odot \rho \in M_{R U C(G)} \subset M_{L_{\infty}(G)}$.

$\operatorname{Pr}$ Proof The fact that $\mathcal{N}\left(L_{p}(G)\right)$ is complete follows from the definition

$\underset{\text { Pruvi }}{\mathcal{N}}\left(L_{p}(G)\right)=L_{p}(G) \otimes_{\gamma} L_{q}(G)$ where $\frac{1}{p}+\frac{1}{q}=1$.

$\mathcal{N}\left(L_{p}(G)\right)=L_{p}(G) \otimes_{\gamma} L_{q}(G)$ where $\frac{1}{p}+\frac{1}{q}=1$.

We first show that for all $\rho, \tau \in \mathcal{N}\left(L_{p}(G)\right), \rho * \tau \in \mathcal{N}\left(L_{p}(G)\right)$ and also the relation $\|\rho * \tau\|_{\mathcal{N}} \leq\|\rho\|_{\mathcal{N}}\|\tau\|_{\mathcal{N}}$ holds. Now, it is clear that $\rho * \tau \in \mathcal{B}\left(L_{p}(G)\right)^{*}$. So, let $\left(T_{\alpha}\right)_{\alpha \in I}$ be a $w^{*}$-convergent net in $\operatorname{Ball}\left(\mathcal{B}\left(L_{p}(G)\right)\right)$ converging to 0 . It suffices to show that $\left\langle\rho * \tau, T_{\alpha}\right\rangle$ converges to 0 in view of Theorem 3.16 in [7]. We have that $\left\langle L_{t} T_{\alpha} L_{t^{-1}}, \rho\right\rangle$ converges to 0 pointwisely and by using the surjection $\pi: \mathcal{N}\left(L_{p}(G)\right) \rightarrow L_{1}(G)$ we have

$$
\begin{aligned}
\left\langle\rho * \tau, T_{\alpha}\right\rangle & =\left\langle\tau, T_{\alpha} \odot \rho\right\rangle \\
& =\left\langle\pi(\tau),\left\langle L_{t} T_{\alpha} L_{t^{-1}}, \rho\right\rangle\right\rangle \\
& =\int_{G}\left\langle L_{t} T_{\alpha} L_{t^{-1}}, \rho\right\rangle \pi(\tau)(t) d \lambda(t)
\end{aligned}
$$

where $\lambda$ is a left Haar measure on $G$. As $\pi(\tau) \in L_{1}(G)$, then $\pi(\tau) d \lambda \in M(G)$. By Theorem 3.1 in [16] (more precisely, part 2. of the proof), this integral goes to 0 in 
$\alpha$. Thus, $\rho * \tau \in \mathcal{N}\left(L_{p}(G)\right)$. To show that $\|\rho * \tau\|_{\mathcal{N}} \leq\|\rho\|_{\mathcal{N}}\|\tau\|_{\mathcal{N}}$ we recall that the canonical embedding of a Banach space into its bidual is isometric. So,

$$
\begin{aligned}
\|\rho * \tau\|_{\mathcal{N}} & =\|\rho * \tau\|_{\mathcal{B}\left(L_{p}(G)\right)^{*}} \\
& =\sup \{|\langle\rho * \tau, T\rangle| ;\|T\| \leq 1\} \\
& =\sup \{|\langle\tau, T \odot \rho\rangle| ;\|T\| \leq 1\} \\
& =\sup \left\{\left|\left\langle\tau, M_{\left\langle L_{t} T L_{t^{-1}}, \rho\right\rangle}\right\rangle\right| ;\|T\| \leq 1\right\} \\
& \leq \sup \left\{\|\tau\|_{\mathcal{N}}\left\|M_{\left\langle L_{t} T L_{t^{-1}}, \rho\right\rangle}\right\| ;\|T\| \leq 1\right\} \\
& \leq \sup \left\{\|\tau\|_{\mathcal{N}}\left|\left\langle L_{t} T L_{t^{-1}}, \rho\right\rangle\right| ;\|T\| \leq 1\right\} \\
& \leq \sup \left\{\|\tau\|_{\mathcal{N}}\left\|L_{t} T L_{t^{-1}}\right\|\|\rho\|_{\mathcal{N}} ;\|T\| \leq 1\right\} \\
& \leq \sup \left\{\|\tau\|_{\mathcal{N}}\left\|L_{t}\right\|\|T\|\left\|L_{t^{-1}}\right\|\|\rho\|_{\mathcal{N}} ;\|T\| \leq 1\right\} \\
& \leq\|\tau\|_{\mathcal{N}}\|\rho\|_{\mathcal{N}} .
\end{aligned}
$$

Next we show that $*$ is distributive and associative. Let $\rho, \tau, \phi \in \mathcal{N}\left(L_{p}(G)\right)$ and $T \in \mathcal{B}\left(L_{p}(G)\right)$.

Distributive: We have

$$
\langle(\rho+\tau) * \phi, T\rangle=\langle\phi, T \odot(\rho+\tau)\rangle
$$

and

$$
\begin{aligned}
T \odot(\rho+\tau) & =M_{\left\langle L_{t} T L_{t^{-1}}, \rho+\tau\right\rangle} \\
& =M_{\left\langle L_{t} T L_{t^{-1}}, \rho\right\rangle+\left\langle L_{t} T L_{t^{-1}}, \tau\right.} \\
& =M_{\left\langle L_{t} T L_{t^{-1}}, \rho\right\rangle}+M_{\left\langle L_{t} T L_{t^{-1}}, \tau\right\rangle} \\
& =T \odot \rho+T \odot \tau .
\end{aligned}
$$


So

$$
\begin{aligned}
\langle(\rho+\tau) * \phi, T\rangle & =\langle\phi, T \odot(\rho+\tau)\rangle \\
& =\langle\phi, T \odot \rho+T \odot \tau\rangle \\
& =\langle\phi, T \odot \rho\rangle+\langle\phi, T \odot \tau\rangle \\
& =\langle\rho * \phi, T\rangle+\langle\tau * \phi, T\rangle \\
& =\langle\rho * \phi+\tau * \phi, T\rangle .
\end{aligned}
$$

Also,

$$
\langle\phi *(\rho+\tau), T\rangle=\langle(\rho+\tau), T \odot \phi\rangle=\langle\rho, T \odot \phi\rangle+\langle\tau, T \odot \phi\rangle=\langle\phi * \rho+\phi * \tau, T\rangle .
$$

Associative: By Proposition 3.1.1 we have that $h(t):=\left\langle L_{t} T L_{t^{-1}}, \rho\right\rangle \in R U C(G)$ and $l_{s} h(t)=h(s t)=\left\langle L_{s t} T L_{(s t)^{-1}}, \rho\right\rangle$. Also, for every $f \in L_{p}(G)$ and $x \in G$,

$$
L_{s} M_{h} L_{s^{-1}} f(x)=M_{h} l_{s^{-1}} f(s x)=h(s x) f(x)=l_{s} h(x) f(x)=M_{l_{s} h} f(x)
$$

which gives $L_{s} M_{h} L_{s^{-1}}=M_{l_{s} h}$. So,

$$
\begin{aligned}
L_{t} T L_{t^{-1}} \odot \rho & =M_{\left\langle L_{s} L_{t} T L_{t^{-1}} L_{s^{-1}}, \rho\right\rangle} \\
& =M_{\left\langle L_{(t s)} T L_{(t s)^{-1}, \rho}\right\rangle} \\
& =M_{l_{t}\left\langle L_{s} T L_{s^{-1}}, \rho\right\rangle} \\
& =L_{t} M_{\left\langle L_{s} T L_{s^{-1}}, \rho\right\rangle} L_{t^{-1}} \\
& =L_{t}(T \odot \rho) L_{t^{-1}} .
\end{aligned}
$$


Thus,

$$
\begin{aligned}
\langle(\rho * \tau) * \phi, T\rangle & =\langle\phi, T \odot(\rho * \tau)\rangle \\
& =\left\langle\phi, M_{\left\langle L_{t} T L_{t^{-1}}, \rho * \tau\right.}\right\rangle \\
& =\left\langle\phi, M_{\left\langle\rho * \tau, L_{t} T L_{t-1}\right\rangle}\right\rangle \\
& =\left\langle\phi, M_{\left\langle\tau, L_{t} T L_{t^{-1}} \odot \rho\right\rangle}\right\rangle \\
& =\left\langle\phi, M_{\left\langle\tau, L_{t} T \odot \rho L_{t^{-1}}\right\rangle}\right\rangle \\
& =\langle\phi,(T \odot \rho) \odot \tau\rangle \\
& =\langle\tau * \phi, T \odot \rho\rangle \\
& =\langle\rho *(\tau * \phi), T\rangle
\end{aligned}
$$

Scalar multiplication: Let $\alpha \in \mathbb{C}$. We have

$$
\begin{aligned}
\langle\alpha(\rho * \tau), T\rangle & =\alpha\langle\rho * \tau, T\rangle \\
& =\alpha\langle\tau, T \odot \rho\rangle \\
& =\langle\alpha \tau, T \odot \rho\rangle \\
& =\langle\rho *(\alpha \tau), T\rangle
\end{aligned}
$$

and

$$
\begin{aligned}
\langle(\alpha \rho) * \tau, T\rangle & =\langle\tau, T \odot(\alpha \rho)\rangle \\
& =\left\langle\tau, M_{\left\langle L_{t} T L_{t^{-1}}, \alpha \rho\right\rangle}\right\rangle \\
& =\left\langle\tau, M_{\alpha\left\langle L_{t} T L_{\left.t^{-1}, \rho\right\rangle}\right\rangle}\right\rangle \\
& =\langle\tau, \alpha(T \odot \rho)\rangle \\
& =\alpha\langle\tau, T \odot \rho\rangle \\
& =\alpha\langle\rho * \tau, T\rangle .
\end{aligned}
$$




\subsection{The first topological centre of $\mathcal{N}\left(L_{p}(G)\right)$}

For this section we will restrict ourselves to second countable, locally compact noncompact groups. We want to show that the convolution algebra of nuclear operators is left strongly Arens irregular, i.e. $Z_{t}^{1}\left(\mathcal{N}\left(L_{p}(G)\right)^{* *}\right)=\mathcal{N}\left(L_{p}(G)\right)$. This follows closely [15].

To show this we cite Theorem 3.2 [17] but we first need to define a map that is used in this theorem. We define the mapping

$$
\begin{aligned}
\Gamma: R U C(G)^{*} & \rightarrow \mathcal{B}\left(\mathcal{B}\left(L_{p}(G)\right)\right) \\
m & \mapsto \Gamma(m)
\end{aligned}
$$

where for $T \in \mathcal{B}\left(L_{p}(G)\right)$ and $\rho \in \mathcal{N}\left(L_{p}(G)\right)$,

$$
\langle\Gamma(m) T, \rho\rangle=m\left(\left\langle L_{t} T L_{t^{-1}}, \rho\right\rangle\right) \equiv\left\langle m,\left\langle L_{t} T L_{t^{-1}}, \rho\right\rangle\right\rangle
$$

where, as usual, $\left\langle L_{t} T L_{t^{-1}}, \rho\right\rangle$ stands for the function $G \ni t \mapsto\left\langle L_{t} T L_{t^{-1}}, \rho\right\rangle$ which is in $R U C(G)$.

Theorem 3.2.1 Let $\left(T_{n}\right)$ be an arbitrary bounded sequence of operators in $\mathcal{B}\left(L_{p}(G)\right)$. Then there exists a sequence $\left(\psi_{n}\right) \subset \operatorname{Ball}\left(R U C(G)^{*}\right)$ and a single operator $T \in \mathcal{B}\left(L_{p}(G)\right)$ such that the factorization $T_{n}=\Gamma\left(\psi_{n}\right) T$ holds for all $n$.

With Theorem 2.2.1 it is enough to show that $\mathcal{N}\left(L_{p}(G)\right)^{*}$ has the left $\mathcal{N}\left(L_{p}(G)\right)^{* *}$ factorization property of level $\aleph_{0}$ and $\mathcal{N}\left(L_{p}(G)\right)$ has the Mazur property of level $\aleph_{0}$. The last condition is true since by Lemma 7.5 of [12], $\mathcal{N}\left(L_{p}(G)\right)=L_{p}(G) \otimes_{\gamma} L_{q}(G)$ is even separable because $G$ is second countable.

As can be seen from the definition of the factorization property we need to have our sequence $\left(\psi_{n}\right)$ in Ball $\left(\mathcal{N}\left(L_{p}(G)\right)^{* *}\right)$. It is easy to make this change and we show this with the next theorem.

Theorem 3.2.2 Let $\left(T_{n}\right)$ be an arbitrary bounded sequence of operators in $\mathcal{N}\left(L_{p}(G)\right)^{*}$. Then there exists a sequence $\left(m_{n}\right) \subset \operatorname{Ball}\left(\mathcal{N}\left(L_{p}(G)\right)^{* *}\right)$ and a single operator $T \in \mathcal{N}\left(L_{p}(G)\right)^{*}$ such that the factorization $T_{n}=m_{n} \odot T$ holds for all $n$. 
Proof Given a sequence $\left(T_{n}\right)_{n \in \mathbb{N}} \subset \mathcal{N}\left(L_{p}(G)\right)^{*}=\mathcal{B}\left(L_{p}(G)\right)$, by Theorem 3.2.1 we have a sequence $\left(\psi_{n}\right) \subset \operatorname{Ball}\left(R U C(G)^{*}\right)$ and an operator $T \in \mathcal{N}\left(L_{p}(G)\right)^{*}$ such that $T_{n}=\Gamma\left(\psi_{n}\right) T$ holds for all $n$. For each $n$, the elements $\psi_{n} \in R U C(G)^{*}$ can be used to define functionals $\psi_{n}^{\prime}$ on $M_{R U C(G)}^{*}$ canonically by the following. For $h \in R U C(G)$ define $\psi_{n}^{\prime} \in M_{R U C(G)}^{*}$ by $\left\langle\psi_{n}^{\prime}, M_{h}\right\rangle:=\left\langle\psi_{n}, h\right\rangle$. Let $m_{n}$ be norm preserving HahnBanach extensions of $\psi_{n}^{\prime}$ to $\mathcal{B}\left(L_{p}(G)\right)^{*}$. Then for all $\rho \in \mathcal{N}\left(L_{p}(G)\right)$ we have

$$
\begin{aligned}
\left\langle T_{n}, \rho\right\rangle & =\left\langle\Gamma\left(\psi_{n}\right) T, \rho\right\rangle \\
& =\left\langle\psi_{n},\left\langle L_{t} T L_{t^{-1}}, \rho\right\rangle\right\rangle \\
& =\left\langle m_{n}, M_{\left\langle L_{t} T L_{t^{-1}}, \rho\right\rangle}\right\rangle \\
& =\left\langle m_{n}, T \odot \rho\right\rangle \\
& =\left\langle m_{n} \odot T, \rho\right\rangle .
\end{aligned}
$$

Thus $T_{n}=m_{n} \odot T$.

Theorem 3.2.3 Let $G$ be a second countable, locally compact, non-compact group. Then $\mathcal{N}\left(L_{p}(G)\right.$ is left strongly Arens irregular.

Proof Theorem 3.2.2 shows that $\mathcal{N}\left(L_{p}(G)\right)^{*}$ has the left $\mathcal{N}\left(L_{p}(G)\right)^{* *}$-factorization property of level $\aleph_{0}$ and so we have the conditions of Theorem 2.2.1, hence $\mathcal{N}\left(L_{p}(G)\right)$ is left strongly Arens irregular.

\subsection{The second topological centre of $\mathcal{N}\left(L_{p}(G)\right)$}

In the previous section, it was shown that $\mathcal{N}\left(L_{p}(G)\right)$ is left strongly Arens irregular. Our goal for this section is to show that $\mathcal{N}\left(L_{p}(G)\right)$ is not right strongly Arens irregular. This is done in [15] but we shall provide the details here.

We will use the following two lemmas to prove this result.

Lemma 3.3.1 Let $G$ be a locally compact group. Then $\left(M_{L_{\infty}(G)}\right)^{\perp} \subset Z_{t}^{2}\left(\mathcal{N}\left(L_{p}(G)\right)^{* *}\right)$. 
Proof Let $\rho \in \mathcal{N}\left(L_{p}(G)\right), T \in \mathcal{N}\left(L_{p}(G)\right)^{*}$ and $n \in \mathcal{N}\left(L_{p}(G)\right)^{* *}$ with $\left(\tau_{\alpha}\right)_{\alpha \in I}$ a net in $\mathcal{N}\left(L_{p}(G)\right)$ such that $w^{*}-\lim _{\alpha} \tau_{\alpha}=n$. Then,

$$
\begin{aligned}
\langle T \diamond n, \rho\rangle & =\langle n, \rho \diamond T\rangle \\
& =w^{*}-\lim _{\alpha}\left\langle\tau_{\alpha}, \rho \diamond T\right\rangle \\
& =w^{*}-\lim _{\alpha}\left\langle\rho \diamond T, \tau_{\alpha}\right\rangle \\
& =w^{*}-\lim _{\alpha}\left\langle T, \tau_{\alpha} * \rho\right\rangle \\
& =w^{*}-\lim _{\alpha}\left\langle T \odot \tau_{\alpha}, \rho\right\rangle .
\end{aligned}
$$

Hence we have that $T \diamond n=w^{*}-\lim _{\alpha} T \odot \tau_{\alpha} \in M_{L_{\infty}(G)}$.

Now let $m \in\left(M_{L_{\infty}(G)}\right)^{\perp} \subset \mathcal{N}\left(L_{p}(G)\right)^{* *}$. We want to show that $m \in Z_{t}^{2}\left(\mathcal{N}\left(L_{p}(G)\right)^{* *}\right)$ and the way in which this is done is to prove that for every $n \in \mathcal{N}\left(L_{p}(G)\right)^{* *}$ we have that $n \odot m=0=n \diamond m$.

So with $T \in \mathcal{N}\left(L_{p}(G)\right)^{*}, n \in \mathcal{N}\left(L_{p}(G)\right)^{* *}$ with $\left(\tau_{\alpha}\right)_{\alpha \in I}$ a net in $\mathcal{N}\left(L_{p}(G)\right)$ such that $w^{*}-\lim _{\alpha} \tau_{\alpha}=n$ we see that

$$
T \diamond n=w^{*}-\lim _{\alpha} T \odot \tau_{\alpha} \in M_{L_{\infty}(G)}
$$

and thus

$$
\langle n \diamond m, T\rangle=\langle m, T \diamond n\rangle=0
$$

So this gives that $n \diamond m=0$.

On the other hand with $T \in \mathcal{N}\left(L_{p}(G)\right)^{*}$ and $\rho \in \mathcal{N}\left(L_{p}(G)\right)$ we have,

$$
\langle n \odot m, T\rangle=\langle n, m \odot T\rangle
$$

and

$$
\langle m \odot T, \rho\rangle=\langle m, T \odot \rho\rangle=0 .
$$

Therefore, $m \odot T=0$ which gives that $n \odot m=0$.

Lemma 3.3.2 Let $G$ be a locally compact, non-compact group. Then

$$
\left(M_{L_{\infty}(G)}\right)^{\perp} \backslash \mathcal{N}\left(L_{p}(G)\right) \neq \varnothing
$$


Proof First recall that $\mathcal{N}\left(L_{p}(G)\right)^{*}=\mathcal{B}\left(L_{p}(G)\right)$ and hence $\mathcal{N}\left(L_{p}(G)\right)^{*}$ is unital. By the Hahn-Banach Separation Theorem there exists an $n \in\left(M_{C_{0}(G)}\right)^{\perp}$ such that $\langle n, 1\rangle=1$ since $G$ is non-compact. Let $P M_{p}(G)$ denote the $w^{*}$-closure of the subalgebra of $\mathcal{B}\left(L_{p}(G)\right)$ generated by the right regular representation of $G$ on $L_{p}(G)$. Take $R \in P M_{p}(G) \backslash M_{L_{\infty}(G)}$ and $m \in\left(M_{L_{\infty}(G)}\right)_{\perp}$ such that $\langle m, R\rangle=1$ (again using the Hahn-Banach Separation Theorem). Set $E=m \odot n \in \mathcal{N}\left(L_{p}(G)\right)^{* *}$. We will show that $E \in\left(M_{L_{\infty}(G)}\right)^{\perp} \backslash \mathcal{N}\left(L_{p}(G)\right)$.

Our first step is to prove that $E \neq 0$. So let $T \in \mathcal{N}\left(L_{p}(G)\right)^{*}$, then

$$
\begin{aligned}
\langle E, T\rangle & =\langle m \odot n, T\rangle \\
& =\langle m, n \odot T\rangle \\
& =\langle n \odot T, m\rangle \\
& =\langle n, T \odot m\rangle \\
& =\left\langle n, M_{\left\langle L_{t} T L_{t^{-1}}, m\right\rangle}\right\rangle .
\end{aligned}
$$

Now, since $R \in P M_{p}(G)$ we have that $R L_{t}=L_{t} R \quad \forall t \in G$ and thus from the above calculation we get

$$
\langle E, R\rangle=\left\langle n, M_{\left\langle L_{t} R L_{t^{-1}}, m\right\rangle}\right\rangle=\left\langle n, M_{\langle R, m\rangle}\right\rangle=\left\langle n, M_{1}\right\rangle=\langle n, 1\rangle=1 .
$$

So $E \neq 0$. To see that $E \in\left(M_{L_{\infty}(G)}\right)^{\perp}$ we let $h \in L_{\infty}(G)$ and again using the calculation above and the fact that $m \in\left(M_{L_{\infty}(G)}\right)_{\perp}$ we get

$$
\left\langle E, M_{h}\right\rangle=\left\langle n, M_{\left\langle L_{t} M_{h} L_{t^{-1}}, m\right\rangle}\right\rangle=\left\langle n, M_{\left\langle M_{l_{t}}, m\right\rangle}\right\rangle=0 .
$$

Finally, to show that $E \notin \mathcal{N}\left(L_{p}(G)\right)$. We shall do this by showing that for any compact operator $K$ we have that $\langle E, K\rangle=0$. So, let $K \in \mathcal{K}\left(L_{p}(G)\right)$. Then from [16] (Lemma 2.5) we have that $\left\langle L_{t} K L_{t^{-1}}, m\right\rangle \in C_{0}(G)$. Thus as $n \in\left(C_{0}(G)\right)^{\perp}$ we have

$$
\langle E, K\rangle=\left\langle n, M_{\left\langle L_{t} K L_{t^{-1}}, m\right\rangle}\right\rangle=0
$$

Theorem 3.3.1 Let $G$ be a locally compact, non-compact group. Then $\mathcal{N}\left(L_{p}(G)\right)$ is not right strongly Arens irregular. 
Proof By the previous two lemmas we get that

$$
\varnothing \neq\left(M_{L_{\infty}(G)}\right)^{\perp} \backslash \mathcal{N}\left(L_{p}(G)\right) \subset Z_{t}^{2}\left(\mathcal{N}\left(L_{p}(G)\right)^{* *}\right) \backslash \mathcal{N}\left(L_{p}(G)\right)
$$

Hence, $\mathcal{N}\left(L_{p}(G)\right) \subsetneq Z_{t}^{2}\left(\mathcal{N}\left(L_{p}(G)\right)\right)$.

Combining this section with the last section we arrive at the conclusion that for any second countable locally compact non-compact group $G, \mathcal{N}\left(L_{p}(G)\right)$ is LSAI bot not RSAI. We would like to understand $Z_{t}^{2}\left(\mathcal{N}\left(L_{p}(G)\right)^{* *}\right)$ better. When we restrict ourselves to discrete groups we have discovered a construction in which we can find the second topological centre. To do this we call upon a construction developed by Dales and Lau in [5]. This is given in the following proposition.

Proposition 3.3.1 Let $(A, *)$ be a Banach algebra and $E$ be a Banach A-bimodule. Then $\mathfrak{A}=A \oplus_{\infty} E$ is a Banach algebra where the product on $\mathfrak{A}$ is as follows:

$$
(a, \phi) \square(b, \tau)=(a * b, a \cdot \tau+\phi \cdot b) \quad \forall a, b \in A \quad \forall \phi, \tau \in E
$$

Proof We start by showing that $\mathfrak{A}$ is an algebra. Let $(a, \phi),(b, \tau),(c, \psi) \in A \oplus E$. Associative:

$$
\begin{aligned}
{[(a, \phi) \square(b, \tau)] \square(c, \psi) } & =(a * b, a \cdot \tau+\phi \cdot b) \square(c, \psi) \\
& =((a * b) * c,(a * b) \cdot \psi+(a \cdot \tau+\phi \cdot b) \cdot c) \\
& =(a *(b * c), a \cdot(b \cdot \psi)+(a \cdot \tau) \cdot c+(\phi \cdot b) \cdot c) \\
& =(a *(b * c), a \cdot(b \cdot \psi)+a \cdot(\tau \cdot c)+\phi \cdot(b * c)) \\
& =(a *(b * c), a \cdot(b \cdot \psi+\tau \cdot c)+\phi \cdot(b * c)) \\
& =(a, \phi) \square(b * c, b \cdot \psi+\tau \cdot c) \\
& =(a, \phi) \square[(b, \tau) \square(c, \psi)] .
\end{aligned}
$$


Distributive:

$$
\begin{aligned}
(a, \phi) \square[(b, \tau)+(c, \psi)] & =(a, \phi) \square(b+c, \tau+\psi) \\
& =(a *(b+c), a \cdot(\tau+\psi)+\phi \cdot(b+c)) \\
& =(a * b+a * c, a \cdot \tau+a \cdot \psi+\phi \cdot b+\phi \cdot c) \\
& =(a * b, a \cdot \tau+\phi \cdot b)+(a * c, a \cdot \psi+\phi \cdot c) \\
& =(a, \phi) \square(b, \tau)+(a, \phi) \square(c, \psi)
\end{aligned}
$$

and

$$
\begin{aligned}
{[(a, \phi)+(b, \tau)] \square(c, \psi) } & =(a+b, \phi+\tau) \square(c, \psi) \\
& =((a+b) * c,(a+b) \cdot \psi+(\phi+\tau) \cdot c) \\
& =(a * c+b * c, a \cdot \psi+b \cdot \psi+\phi \cdot c+\tau \cdot c) \\
& =(a * c, a \cdot \psi+\phi \cdot c)+(b * c, b \cdot \psi+\tau \cdot c) \\
& =(a, \phi) \square(c, \psi)+(b, \tau) \square(c, \psi) ;
\end{aligned}
$$

and finally for $\alpha \in \mathbb{C}$

$$
\begin{aligned}
(\alpha(a, \phi)) \square(b, \tau) & =(\alpha a, \alpha \phi) \square(b, \tau) \\
& =((\alpha a) * b,(\alpha a) \cdot \tau+(\alpha \phi) \cdot b) \\
& =(\alpha(a * b), \alpha(a \cdot \tau)+\alpha(\phi \cdot b)) \\
& =\alpha(a * b, a \cdot \tau+\phi \cdot b) \\
& =\alpha((a, \phi) \square(b, \tau)) \\
& =\alpha(a * b, a \cdot \tau+\phi \cdot b) \\
& =(\alpha(a * b), \alpha(a \cdot \tau)+\alpha(\phi \cdot b)) \\
& =(a *(\alpha b), a \cdot(\alpha \tau)+\phi \cdot(\alpha b)) \\
& =(a, \phi) \square(\alpha b, \alpha \tau) \\
& =(a, \phi) \square(\alpha(b, \tau)) .
\end{aligned}
$$


Our goal here is to determine the second topological centre of $\mathcal{N}\left(\ell_{p}(G)\right)^{* *}$, where $G$ is discrete. In the previous section we showed that the first topological centre of $\mathcal{N}\left(\ell_{p}(G)\right)^{* *}$ is $\mathcal{N}\left(\ell_{p}(G)\right)$. In this section we showed that $\mathcal{N}\left(\ell_{p}(G)\right)$ is not right strongly Arens irregular, and indeed we will show that the second topological centre is

$\ell_{1}(G) \oplus\left(M_{\ell_{\infty}(G)}\right)^{\perp}$ while $\mathcal{N}\left(\ell_{p}(G)\right)$ itself is $\ell_{1}(G) \oplus\left(M_{\ell_{\infty}(G)}\right)_{\perp}$.

Proposition 3.3.2 $\mathcal{N}\left(\ell_{p}(G)\right)=\ell_{1}(G) \oplus\left(M_{l_{\infty}(G)}\right)_{\perp}$ as Banach spaces.

Proof We first begin by noting that since $G$ is discrete we can represent an element in $\mathcal{N}\left(\ell_{p}(G)\right)$ as a matrix where the coordinates of the matrix are determined using the group elements. With this representation, one can view $\ell_{1}(G)$ functions as operators in which the matrix representation is a diagonal matrix in a canonical way. This shows that $\ell_{1}(G) \subset \mathcal{N}\left(\ell_{p}(G)\right)$. Now, let $f \in \mathcal{N}\left(\ell_{p}(G)\right)$ and consider its canonical embedding into $\mathcal{N}\left(\ell_{p}(G)\right)^{* *}=\mathcal{B}\left(\ell_{p}(G)\right)^{*}$. Let $f_{1}=\left.f\right|_{\ell_{\infty}(G)} \in \ell_{\infty}(G)^{*}$ (using the natural isometric isomorphism between $\ell_{\infty}(G)$ and $\left.M_{\ell_{\infty}(G)}\right)$. Since $f \in \mathcal{N}\left(\ell_{p}(G)\right)$, $f$ is $w^{*}$ continuous and thus $f_{1}$ is $w^{*}$ continuous which implies that $f_{1} \in \ell_{1}(G)$. Set $f_{2}=f-f_{1}$. We claim that $f_{2} \in\left(M_{\ell_{\infty}(G)}\right)_{\perp}$. Indeed, let $T \in M_{\ell_{\infty}(G)}$ then $T=M_{h}$ for some $h \in \ell_{\infty}(G)$ and so we have

$$
\begin{aligned}
\left\langle T, f_{2}\right\rangle & =\left\langle M_{h}, f_{2}\right\rangle=\left\langle\left. f_{2}\right|_{\ell_{\infty}(G)}, h\right\rangle=\left\langle\left.\left(f-f_{1}\right)\right|_{\ell_{\infty}(G)}, h\right\rangle \\
& =\left\langle\left. f\right|_{\ell_{\infty}(G)}, h\right\rangle-\left\langle f_{1}, h\right\rangle=\left\langle f_{1}, h\right\rangle-\left\langle f_{1}, h\right\rangle=0
\end{aligned}
$$

which gives $\mathcal{N}\left(\ell_{p}(G)\right)=\ell_{1}(G)+\left(M_{\ell_{\infty}(G)}\right)_{\perp}$. As $\ell_{1}(G) \cap\left(M_{\ell_{\infty}(G)}\right)_{\perp}=\{0\}$ we have that this sum is direct.

We now show that $\left(M_{\ell_{\infty}(G)}\right)_{\perp}$ is an ideal in $\mathcal{N}\left(\ell_{p}(G)\right)$. Once we have this property then we can define the action of $\ell_{1}(G)$ on $\left(M_{\ell_{\infty}(G)}\right)_{\perp}$ to be the convolution product on $\mathcal{N}\left(\ell_{p}(G)\right)$ making $\left(M_{\ell_{\infty}(G)}\right)_{\perp}$ a Banach $\ell_{1}(G)$-bimodule.

Lemma 3.3.3 $\mathcal{N}\left(\ell_{p}(G)\right) *\left(M_{\ell_{\infty}(G)}\right)_{\perp}=0$ 
Proof Let $\rho \in \mathcal{N}\left(\ell_{p}(G)\right), \tau \in\left(M_{\ell_{\infty}(G)}\right)_{\perp}$ and $S \in \mathcal{B}\left(\ell_{p}(G)\right)$. Then

$$
\begin{aligned}
\langle\rho * \tau, S\rangle & =\langle\tau, S \odot \rho\rangle \\
& =\left\langle\tau, M_{\left\langle\rho, L_{t} S L_{t^{-1}}\right\rangle}\right\rangle \\
& =0 .
\end{aligned}
$$

Lemma 3.3.4 $\left(M_{\ell_{\infty}(G)}\right)_{\perp} * \mathcal{N}\left(\ell_{p}(G)\right) \subset\left(M_{\ell_{\infty}(G)}\right)_{\perp}$.

Proof Let $\rho \in \mathcal{N}\left(\ell_{p}(G)\right), \tau \in\left(M_{\ell_{\infty}(G)}\right)_{\perp}, T \in M_{\ell_{\infty}(G)}$. We want to show that $\langle\tau * \rho, T\rangle=0$. We have $T=M_{h}$ for some $h \in \ell_{\infty}(G)$. Recall that $L_{t} M_{h} L_{t^{-1}}=M_{l_{t} h}$. Thus

$$
\langle\tau * \rho, T\rangle=\langle\rho, T \odot \tau\rangle=0
$$

since

$$
T \odot \tau=M_{\left\langle L_{t} T L_{t^{-1}}, \tau\right\rangle}=M_{\left\langle M_{l_{t}}, \tau\right\rangle}=0 .
$$

Remark: The last two lemmas hold for any arbitrary locally compact group but for our purpose here, we need only the discrete case.

So, combining the last two lemmas we see that $\left(M_{\ell_{\infty}(G)}\right)_{\perp}$ is an ideal in $\mathcal{N}\left(\ell_{p}(G)\right)$. We continue by linking the product on $\mathcal{N}\left(\ell_{p}(G)\right)$ to the product $\square$ on $\ell_{1}(G) \oplus\left(M_{\ell_{\infty}(G)}\right)_{\perp}$.

Proposition 3.3.3 $\left(\ell_{1}(G) \oplus\left(M_{l_{\infty}(G)}\right)_{\perp}, \square\right)=\left(\mathcal{N}\left(\ell_{p}(G)\right), *\right)$, where the product $\square$ is defined by

$$
(a, x) \square(b, y)=(a * b, a * y+x * b) \quad\left(a, b \in \ell_{1}(G), x, y \in\left(M_{l_{\infty}(G)}\right)_{\perp}\right) .
$$

Proof By Proposition 3.3.2, $\mathcal{N}\left(\ell_{p}(G)\right)=\ell_{1}(G) \oplus\left(M_{l_{\infty}(G)}\right)_{\perp}$, so for $\phi, \lambda \in \mathcal{N}\left(\ell_{p}(G)\right)$ there are $\phi_{1}, \lambda_{1} \in \ell_{1}(G)$ and $\phi_{2}, \lambda_{2} \in\left(M_{\ell_{\infty}(G)}\right)_{\perp}$ such that $\phi=\phi_{1}+\phi_{2}$ and $\lambda=\lambda_{1}+\lambda_{2}$. Let $\theta$ be the isomorphism between $\mathcal{N}\left(\ell_{p}(G)\right)$ and $\ell_{1}(G) \oplus\left(M_{\ell_{\infty}(G)}\right)_{\perp}$, i.e., $\theta\left(\phi_{1}, \phi_{2}\right)=\phi_{1}+\phi_{2}=\phi$. We now check that $\phi * \lambda=\theta\left(\left(\phi_{1}, \phi_{2}\right) \square\left(\lambda_{1}, \lambda_{2}\right)\right)$. 
Case $1\left(\lambda \in \ell_{1}(G)\right)$ : Here we have $\phi=\phi_{1}+\phi_{2}$ and $\lambda=\lambda_{1}$. We obtain

$$
\phi * \lambda=\left(\phi_{1}+\phi_{2}\right) * \lambda_{1}=\phi_{1} * \lambda_{1}+\phi_{2} * \lambda_{1}
$$

and

$$
\begin{aligned}
\theta\left(\left(\phi_{1}, \phi_{2}\right) \square\left(\lambda_{1}, 0\right)\right) & =\theta\left(\phi_{1} * \lambda_{1}, \phi_{1} * 0+\phi_{2} * \lambda_{1}\right) \\
& =\theta\left(\phi_{1} * \lambda_{1}, \phi_{2} * \lambda_{1}\right) \\
& =\phi_{1} * \lambda_{1}+\phi_{2} * \lambda_{1} \\
& =\phi * \lambda .
\end{aligned}
$$

Case $2\left(\lambda \in\left(M_{\ell_{\infty}(G)}\right)_{\perp}\right)$ : Here we have $\phi=\phi_{1}+\phi_{2}$ and $\lambda=\lambda_{2}$ and in this case by Lemma 3.3.3 we have that $\phi * \lambda=0$. On the other hand using again Lemma 3.3.3,

$$
\begin{aligned}
\theta\left(\left(\phi_{1}, \phi_{2}\right) \square\left(0, \lambda_{2}\right)\right) & =\theta\left(\phi_{1} * 0, \phi_{1} * \lambda_{2}+\phi_{2} * 0\right) \\
& =\theta\left(0, \phi_{1} * \lambda_{2}\right) \\
& =\theta(0,0) \\
& =0 \\
& =\phi * \lambda .
\end{aligned}
$$

Since $\square$ is distributive and $\left(\lambda_{1}, \lambda_{2}\right)=\left(\lambda_{1}, 0\right)+\left(0, \lambda_{2}\right)$ it is now clear that

$$
\phi * \lambda=\theta\left(\left(\phi_{1}, \phi_{2}\right) \square\left(\lambda_{1}, \lambda_{2}\right)\right) \quad \forall \phi, \lambda \in \mathcal{N}\left(\ell_{p}(G)\right)
$$

Now that we have an alternate description of $\mathcal{N}\left(\ell_{p}(G)\right)$, we need to determine how the product $\square$ extends to the bidual level. We point out here that we extend to the bidual level given the specific actions of $A$ on $E$ above; note that in [10], M. Eshaghi Gordji and M. Filali show how one extends a module action to the bidual level in full generality.

Note that if $\mathfrak{A}=A \oplus E$ then $\mathfrak{A}^{* *}=A^{* *} \oplus E^{* *}$.

Proposition 3.3.4 Let $\left(\Phi_{1}, \Phi_{2}\right),\left(\Lambda_{1}, \Lambda_{2}\right) \in \ell_{1}(G)^{* *} \oplus\left(M_{\ell_{\infty}(G)}\right)_{\perp}^{* *}$. Then

$$
\left(\Phi_{1}, \Phi_{2}\right) \diamond\left(\Lambda_{1}, \Lambda_{2}\right)=\left(\Phi_{1} \diamond \Lambda_{1}, \Phi_{1} \diamond \Lambda_{2}+\Phi_{2} \diamond \Lambda_{1}\right)
$$


Proof Fix $\left(\lambda_{1}^{(0)}, \lambda_{2}^{(0)}\right) \in \ell_{1}(G) \oplus\left(M_{\ell_{\infty}(G)}\right)_{\perp}$ and let $\left(\Phi_{1}, \Phi_{2}\right) \in \ell_{1}(G)^{* *} \oplus\left(M_{\ell_{\infty}(G)}\right)_{\perp}^{* *}$. Take $\left(\phi_{1}^{(\alpha)}, \phi_{2}^{(\alpha)}\right)_{\alpha \in I} \subset \ell_{1}(G) \oplus\left(M_{\ell_{\infty}(G)}\right)_{\perp}$ such that $w^{*}-\lim _{\alpha}\left(\phi_{1}^{(\alpha)}, \phi_{2}^{(\alpha)}\right)=\left(\Phi_{1}, \Phi_{2}\right)$ for some index set $I$. Then

$$
\begin{aligned}
& \left(\Phi_{1}, \Phi_{2}\right) \diamond\left(\lambda_{1}^{(0)}, \lambda_{2}^{(0)}\right) \\
= & \left(w^{*}-\lim _{\alpha}\left(\phi_{1}^{(\alpha)}, \phi_{2}^{(\alpha)}\right)\right) \diamond\left(\lambda_{1}^{(0)}, \lambda_{2}^{(0)}\right) \\
= & w^{*}-\lim _{\alpha}\left(\left(\phi_{1}^{(\alpha)}, \phi_{2}^{(\alpha)}\right) \diamond\left(\lambda_{1}^{(0)}, \lambda_{2}^{(0)}\right)\right) \\
= & w^{*}-\lim _{\alpha}\left(\left(\phi_{1}^{(\alpha)}, \phi_{2}^{(\alpha)}\right) \square\left(\lambda_{1}^{(0)}, \lambda_{2}^{(0)}\right)\right) \\
= & w^{*}-\lim _{\alpha}\left(\phi_{1}^{(\alpha)} * \lambda_{1}^{(0)}, \phi_{1}^{(\alpha)} * \lambda_{2}^{(0)}+\phi_{2}^{(\alpha)} * \lambda_{1}^{(0)}\right) \\
= & w^{*}-\lim _{\alpha}\left(\phi_{1}^{(\alpha)} \diamond \lambda_{1}^{(0)}, \phi_{1}^{(\alpha)} \diamond \lambda_{2}^{(0)}+\phi_{2}^{(\alpha)} \diamond \lambda_{1}^{(0)}\right) \\
= & \left(w^{*}-\lim _{\alpha} \phi_{1}^{(\alpha)} \diamond \lambda_{1}^{(0)}, w^{*}-\lim _{\alpha} \phi_{1}^{(\alpha)} \diamond \lambda_{2}^{(0)}+w^{*}-\lim _{\alpha} \phi_{2}^{(\alpha)} \diamond \lambda_{1}^{(0)}\right) \\
= & \left(\Phi_{1} \diamond \lambda_{1}^{(0)}, \Phi_{1} \diamond \lambda_{2}^{(0)}+\Phi_{2} \diamond \lambda_{1}^{(0)}\right) .
\end{aligned}
$$

Now take $\left(\Lambda_{1}, \Lambda_{2}\right) \in \ell_{1}(G)^{* *} \oplus\left(M_{\ell_{\infty}(G)}\right)_{\perp}^{* *}$ and $\left(\lambda_{1}^{(\alpha)}, \lambda_{2}^{(\alpha)}\right)_{\alpha \in I} \subset \ell_{1}(G) \oplus\left(M_{\ell_{\infty}(G)}\right)_{\perp}$ such that $w^{*}-\lim _{\alpha}\left(\lambda_{1}^{(\alpha)}, \lambda_{2}^{(\alpha)}\right)=\left(\Lambda_{1}, \Lambda_{2}\right)$. Then

$$
\begin{aligned}
& \left(\Phi_{1}, \Phi_{2}\right) \diamond\left(\Lambda_{1}, \Lambda_{2}\right) \\
= & \left(\Phi_{1}, \Phi_{2}\right) \diamond\left(w^{*}-\lim _{\alpha}\left(\lambda_{1}^{(\alpha)}, \lambda_{2}^{(\alpha)}\right)\right) \\
= & w^{*}-\lim _{\alpha}\left(\left(\Phi_{1}, \Phi_{2}\right) \diamond\left(\lambda_{1}^{(\alpha)}, \lambda_{2}^{(\alpha)}\right)\right) \\
= & w^{*}-\lim _{\alpha}\left(\Phi_{1} \diamond \lambda_{1}^{(\alpha)}, \Phi_{1} \diamond \lambda_{2}^{(\alpha)}+\Phi_{2} \diamond \lambda_{1}^{(\alpha)}\right) \\
= & \left(w^{*}-\lim _{\alpha} \Phi_{1} \diamond \lambda_{1}^{(\alpha)}, w^{*}-\lim _{\alpha} \Phi_{1} \diamond \lambda_{2}^{(\alpha)}+w^{*}-\lim _{\alpha} \Phi_{2} \diamond \lambda_{1}^{(\alpha)}\right) \\
= & \left(\Phi_{1} \diamond \Lambda_{1}, \Phi_{1} \diamond \Lambda_{2}+\Phi_{2} \diamond \Lambda_{1}\right) .
\end{aligned}
$$

Using the previous proposition we are now ready to calculate the second topological centre of $\mathcal{N}\left(\ell_{p}(G)\right)^{* *}$. 
Theorem 3.3.2 $Z_{t}^{2}\left(\mathcal{N}\left(\ell_{p}(G)\right)^{* *}\right)=\ell_{1}(G) \oplus\left(M_{\ell_{\infty}(G)}\right)^{\perp}$.

Proof We start by showing that $\Psi \diamond \Lambda=0 \forall \Psi \in \ell_{1}(G)^{* *}, \Lambda \in\left(M_{\ell_{\infty}(G)}\right)_{\perp}^{* *}$. Indeed, by Lemma 3.3 .3 we have that $\ell_{1}(G) *\left(M_{\ell_{\infty}(G)}\right)_{\perp}=0$. Fix $\lambda_{0} \in\left(M_{\ell_{\infty}(G)}\right)_{\perp}$ and let $\Psi \in \ell_{1}(G)^{* *}$ and $\left(\psi_{\alpha}\right)_{\alpha \in I} \subset \ell_{1}(G)$ such that $\Psi=w^{*}-\lim _{\alpha} \psi_{\alpha}$ for some index set $I$. Then we have

$$
\Psi \diamond \lambda_{0}=\left(w^{*}-\lim _{\alpha} \psi_{\alpha}\right) \diamond \lambda_{0}=w^{*}-\lim _{\alpha}\left(\psi_{\alpha} \diamond \lambda_{0}\right)=w^{*}-\lim _{\alpha}\left(\psi_{\alpha} * \lambda_{0}\right)=0
$$

Now take $\Lambda \in\left(M_{\ell_{\infty}(G)}\right)_{\perp}^{* *}$ and $\left(\lambda_{\alpha}\right)_{\alpha \in J} \subset\left(M_{\ell_{\infty}(G)}\right)_{\perp}$ such that $\Lambda=w^{*}-\lim _{\alpha} \lambda_{\alpha}$ for some index set $J$. Then

$$
\Psi \diamond \Lambda=\Psi \diamond\left(w^{*}-\lim _{\alpha} \lambda_{\alpha}\right)=w^{*}-\lim _{\alpha}\left(\Psi \diamond \lambda_{\alpha}\right)=0 .
$$

So, let $(\Phi, \Lambda) \in Z_{t}^{2}\left(\ell_{1}(G)^{* *} \oplus\left(M_{\ell_{\infty}(G)}\right)_{\perp}^{* *}\right)$ and take a net $\left(\mu_{\alpha}, \nu_{\alpha}\right)$ in $\ell_{1}(G)^{* *} \oplus\left(M_{\ell_{\infty}(G)}\right)_{\perp}^{* *}$ which converges $w^{*}$ to 0 . Then by Proposition 3.3.4 we have that for each $\alpha$

$$
\left(\mu_{\alpha}, \nu_{\alpha}\right) \diamond(\Phi, \Lambda)=\left(\mu_{\alpha} \diamond \Phi, \mu_{\alpha} \diamond \Lambda+\nu_{\alpha} \diamond \Phi\right)=\left(\mu_{\alpha} \diamond \Phi, \nu_{\alpha} \diamond \Phi\right)
$$

Now, since $(\Phi, \Lambda) \in Z_{t}^{2}\left(\ell_{1}(G)^{* *} \oplus\left(M_{\ell_{\infty}(G)}\right)_{\perp}^{* *}\right)$, both $\mu_{\alpha} \diamond \Phi$ and $\nu_{\alpha} \diamond \Phi$ converge $w^{*}$ to 0 . Thus $\Phi \in Z_{t}^{2}\left(\ell_{1}(G)^{* *}\right)=\ell_{1}(G)$ and so

$$
Z_{t}^{2}\left(\ell_{1}(G)^{* *} \oplus\left(M_{\ell_{\infty}(G)}\right)_{\perp}^{* *}\right) \subset \ell_{1}(G) \oplus\left(M_{\ell_{\infty}(G)}\right)_{\perp}^{* *}
$$

By Lemma 3.3.1 we have $\left(M_{\ell_{\infty}(G)}\right)^{\perp} \subset Z_{t}^{2}\left(\mathcal{N}\left(\ell_{p}(G)\right)\right.$ which implies that

$$
\ell_{1}(G) \oplus\left(M_{\ell_{\infty}(G)}\right)^{\perp} \subset Z_{t}^{2}\left(\ell_{1}^{* *} \oplus\left(M_{\ell_{\infty}(G)}\right)_{\perp}^{* *}\right)
$$

It remains to show that $\left(M_{\ell_{\infty}(G)}\right)_{\perp}^{* *}=\left(M_{\ell_{\infty}(G)}\right)^{\perp}$. By Proposition 2.6.6 in [14] we have that $\left(\left(M_{\ell_{\infty}(G)}\right)_{\perp \perp}\right)^{\perp}={\overline{\left(M_{\ell_{\infty}}(G)\right)_{\perp}}}^{w *}$ and since ${\overline{\left(M_{\ell_{\infty}}(G)\right)_{\perp}}}^{w *}=\left(M_{\ell_{\infty}(G)}\right)_{\perp}^{* *}$ it suffices to show that $\left(M_{\ell_{\infty}(G)}\right)_{\perp \perp}=M_{\ell_{\infty}(G)}$. Now, as

$$
\left(M_{\ell_{\infty}(G)}\right)_{\perp \perp}=\left\{T \in \mathcal{B}\left(\ell_{p}(G)\right) \mid\langle T, \rho\rangle=0 \quad \forall \rho \in\left(M_{\ell_{\infty}(G)}\right)_{\perp}\right\}
$$

it is clear that $M_{\ell_{\infty}(G)} \subset\left(M_{\ell_{\infty}(G)}\right)_{\perp \perp}$. For the opposite inclusion let $\phi \in\left(M_{\ell_{\infty}(G)}\right)_{\perp \perp}$. Then $\left.\phi\right|_{\left(M_{\ell_{\infty}(G)}\right)_{\perp}} \equiv 0$ which implies that $\phi \in\left(\left(M_{\ell_{\infty}(G)}\right)_{\perp}\right)^{\perp}$. Again, by Proposition 2.6.6 in [14], $\left(\left(M_{\ell_{\infty}(G)}\right)_{\perp}\right)^{\perp}={\overline{M_{\ell_{\infty}}(G)}}^{w^{*}} \subset \mathcal{N}\left(\ell_{p}(G)\right)^{*}$. As $M_{\ell_{\infty}(G)}$ is $w^{*}$-closed, we get the desired inclusion. 


\section{Chapter 4}

\section{Strong Arens irregularity is not hereditary}

In the last section we showed that $\mathcal{N}\left(\ell_{p}(G)\right)$ is LSAI but not RSAI and it is clear

that $\left(M_{l_{\infty(G)}}\right)_{\perp}$ is a subalgebra that is Arens regular. So we have already provided an example of a Banach algebra with a subalgebra that has a different topological centre. In this section we will construct a Banach algebra that is SAI which has a subalgebra that is non-trivially Arens regular (non-trivial in the sense that it is not SAI) where we use the example of $\mathcal{N}\left(\ell_{p}(G)\right)$ as motivation.

\subsection{Strongly Arens irregular Banach algebras with an Arens regular subalgebra}

Since any finite dimensional Banach algebra is reflexive it is trivially true that every strongly Arens irregular Banach algebra has a subalgebra that is Arens regular. Our goal here is to construct an algebra that is SAI that admits a subalgebra that is AR and not SAI.

Recall that if $A$ is a Banach algebra and $E$ is a Banach $A$-bimodule then $A \oplus E$ is a Banach algebra where the product is

$$
(a, x) \square(b, y)=(a b, a \cdot y+x \cdot b) \quad \forall(a, x),(b, y) \in A \oplus E .
$$


Note that for $G$ discrete we have that $\mathcal{N}\left(\ell_{p}(G)\right)=\ell_{1}(G) \oplus\left(M_{\ell_{\infty}(G)}\right)_{\perp}$ and by Proposition 3.3.2 we have that $\left(\mathcal{N}\left(\ell_{p}(G)\right), *\right)=\left(\ell_{1}(G) \oplus\left(M_{\ell_{\infty}(G)}\right)_{\perp}, \square\right)$. The algebra $\mathcal{N}\left(\ell_{p}(G)\right)$ is close to our goal since it is left strongly Arens irregular with a subalgebra $\left(M_{\ell_{\infty}(G)}\right)_{\perp}$ that is Arens regular. Our construction is modeled after the structure of $\ell_{1}(G) \oplus\left(M_{\ell_{\infty}(G)}\right)_{\perp}$. The next few propositions will give us the tools needed to construct the desired algebra.

Proposition 4.1.1 Let $A$ be a commutative Banach algebra and let $E$ be a Banach algebra that is also a Banach A-bimodule. If - denotes an action of $A$ on $E$ from the left then

$$
x \cdot{ }_{r} a=a \cdot x \quad \forall a \in A, x \in E
$$

defines an action of $A$ on $E$ from the right.

Proof Let $f, g \in A$ and $\tau \in E$. Then

$$
\begin{aligned}
(f g) \cdot{ }_{r} \tau & =\tau \cdot(f g) \\
& =\tau \cdot(g f) \\
& =(\tau \cdot g) \cdot f \\
& =f \cdot{ }_{r}(\tau \cdot g) \\
& =f \cdot{ }_{r}\left(g \cdot{ }_{r} \tau\right) .
\end{aligned}
$$

The other properties are trivial.

Proposition 4.1.2 Let $A \oplus E$ be as in the above proposition. Let $\left(\Phi_{1}, \Lambda_{1}\right),\left(\Phi_{2}, \Lambda_{2}\right) \in A^{* *} \oplus E^{* *}$. Then

$$
\left(\Phi_{1}, \Lambda_{1}\right) \odot\left(\Phi_{2}, \Lambda_{2}\right)=\left(\Phi_{1} \odot \Phi_{2}, \Lambda_{2} \diamond \Phi_{1}+\Lambda_{1} \odot \Phi_{2}\right)
$$

and

$$
\left(\Phi_{1}, \Lambda_{1}\right) \diamond\left(\Phi_{2}, \Lambda_{2}\right)=\left(\Phi_{1} \diamond \Phi_{2}, \Lambda_{2} \odot \Phi_{1}+\Lambda_{1} \diamond \Phi_{2}\right)
$$

Here $\odot$ and $\diamond$ are used to denote the extensions of the module actions in a similar way as the product on $A$. 
Proof Fix $\left(\phi_{1}, \lambda_{1}\right) \in A \oplus E$ and let $\left(\Phi_{2}, \Lambda_{2}\right) \in A^{* *} \oplus E^{* *}$. Take $\left(\phi_{2}^{(\alpha)}, \lambda_{2}^{(\alpha)}\right)_{\alpha \in I} \subset A \oplus E$ such that $w^{*}-\lim _{\alpha}\left(\phi_{2}^{(\alpha)}, \lambda_{2}^{(\alpha)}\right)=\left(\Phi_{2}, \Lambda_{2}\right)$ for some index set $I$. Then

$$
\begin{aligned}
& \left(\phi_{1}, \lambda_{1}\right) \odot\left(\Phi_{2}, \Lambda_{2}\right) \\
& =\left(\phi_{1}, \lambda_{1}\right) \odot\left(w^{*}-\lim _{\alpha}\left(\phi_{2}^{(\alpha)}, \lambda_{2}^{(\alpha)}\right)\right) \\
& =w^{*}-\lim _{\alpha}\left(\left(\phi_{1}, \lambda_{1}\right) \odot\left(\phi_{2}^{(\alpha)}, \lambda_{2}^{(\alpha)}\right)\right) \\
& =w^{*}-\lim _{\alpha}\left(\left(\phi_{1}, \lambda_{1}\right)\left(\phi_{2}^{(\alpha)}, \lambda_{2}^{(\alpha)}\right)\right) \\
& =w^{*}-\lim _{\alpha}\left(\phi_{1} * \phi_{2}^{(\alpha)}, \phi_{1} \cdot \lambda_{2}^{(\alpha)}+\lambda_{1} \cdot \phi_{2}^{(\alpha)}\right) \\
& =w^{*}-\lim _{\alpha}\left(\phi_{1} * \phi_{2}^{(\alpha)}, \lambda_{2}^{(\alpha)} * \phi_{1}+\lambda_{1} * \phi_{2}^{(\alpha)}\right) \\
& =w^{*}-\lim _{\alpha}\left(\phi_{1} \odot \phi_{2}^{(\alpha)}, \lambda_{2}^{(\alpha)} \diamond \phi_{1}+\lambda_{1} \odot \phi_{2}^{(\alpha)}\right) \\
& =\left(w^{*}-\lim _{\alpha} \phi_{1} \odot \phi_{2}^{(\alpha)}, w^{*}-\lim _{\alpha} \lambda_{2}^{(\alpha)} \diamond \phi_{1}+w^{*}-\lim _{\alpha} \lambda_{1} \odot \phi_{2}^{(\alpha)}\right) \\
& =\left(\phi_{1} \odot \Phi_{2}, \Lambda_{2} \diamond \phi_{1}+\lambda_{1} \odot \Phi_{2}\right) .
\end{aligned}
$$

Now take $\left(\Phi_{1}, \Lambda_{1}\right) \in \ell_{1}(G)^{* *} \oplus\left(M_{\ell_{\infty}(G)}\right)_{\perp}^{* *}$ and $\left(\phi_{1}^{(\alpha)}, \lambda_{1}^{(\alpha)}\right)_{\alpha \in I} \subset \ell_{1}(G) \oplus\left(M_{\ell_{\infty}(G)}\right)_{\perp}$ such that $w^{*}-\lim _{\alpha}\left(\phi_{1}^{(\alpha)}, \lambda_{1}^{(\alpha)}\right)=\left(\Phi_{1}, \Lambda_{1}\right)$. Then

$$
\begin{aligned}
& \left(\Phi_{1}, \Lambda_{1}\right) \odot\left(\Phi_{2}, \Lambda_{2}\right) \\
& =\left(w^{*}-\lim _{\alpha}\left(\phi_{1}^{(\alpha)}, \lambda_{1}^{(\alpha)}\right)\right) \odot\left(\Phi_{2}, \Lambda_{2}\right) \\
& =w^{*}-\lim _{\alpha}\left(\left(\phi_{1}^{(\alpha)}, \lambda_{1}^{(\alpha)}\right) \odot\left(\Phi_{2}, \Lambda_{2}\right)\right) \\
& =w^{*}-\lim _{\alpha}\left(\phi_{1}^{(\alpha)} \odot \Phi_{2}, \Lambda_{2} \diamond \phi_{1}^{(\alpha)}+\lambda_{1}^{(\alpha)} \odot \Phi_{2}\right) \\
& =\left(w^{*}-\lim _{\alpha} \phi_{1}^{(\alpha)} \odot \Phi_{2}, w^{*}-\lim _{\alpha} \Lambda_{2} \diamond \phi_{1}^{(\alpha)}+w^{*}-\lim _{\alpha} \lambda_{1}^{(\alpha)} \odot \Phi_{2}\right) \\
& =\left(\Phi_{1} \odot \Phi_{2}, \Lambda_{2} \diamond \Phi_{1}+\Lambda_{1} \odot \Phi_{2}\right) .
\end{aligned}
$$

Similar calculations show that the second equation holds, where first we fix $\left(\phi_{2}, \lambda_{2}\right) \in A \oplus E$ and approximate $\left(\Phi_{1}, \Lambda_{1}\right) \in A^{* *} \oplus E^{* *}$ with a net in $A \oplus E$ to obtain

$$
\left(\Phi_{1}, \Lambda_{1}\right) \diamond\left(\phi_{2}, \lambda_{2}\right)=\left(\Phi_{1} \diamond \phi_{2}, \lambda_{2} \odot \Phi_{1}+\Lambda_{1} \diamond \phi_{2}\right)
$$

Then we approximate $\left(\Phi_{2}, \Lambda_{2}\right) \in A^{* *} \oplus E^{* *}$ with a net in $A \oplus E$ to get the desired result.

We are now ready to give a classification of the topological centres of $\mathfrak{A}$. 
Lemma 4.1.1 ([9]) Given $(\mathfrak{A}=A \oplus E, \square)$ then the first topological centre is given by all the elements of the form $(\Phi, \Lambda) \in \mathfrak{A}^{* *}$ such that the following hold:

(1) $\Phi \in Z_{t}^{1}\left(A^{* *}\right)$;

(2) the map $E^{* *} \ni N \mapsto \Phi \cdot N$ is $w^{*}-w^{*}$ continuous;

(3) the map $A^{* *} \ni M \mapsto \Lambda \cdot M$ is $w^{*}-w^{*}$ continuous.

Proof Let $(\Phi, \Lambda) \in Z_{t}^{1}\left(\mathfrak{A}^{* *}\right)$ and take $\left(\mu_{\alpha}, \nu_{\alpha}\right)$ a net in $\mathfrak{A}^{* *}$ converging $w^{*}$ to zero. This implies that $(\Phi, \Lambda) \odot\left(\mu_{\alpha}, \nu_{\alpha}\right)=\left(\Phi \odot \mu_{\alpha}, \Phi \cdot \nu_{\alpha}+\Lambda \cdot \mu_{\alpha}\right)$ converges $w^{*}$ to zero. It is clear that $(1)$ holds and by taking the net $\left(0, \nu_{\alpha}\right)$ we must obtain the same result which implies that (2) holds. We also know by taking the net $\left(\mu_{\alpha}, 0\right)$ that $\Lambda \cdot \mu_{\alpha}$ converges $w^{*}$ to zero which gives (3). The converse is trivial.

Theorem 4.1.1 Let $A$ be a commutative Banach algebra that is strongly Arens irregular. Let $E$ be a Banach space such that $\left|E^{* *}\right|>|E|$ and such that $E$ is also a Banach $A$-bimodule. Define the product on $E$ to be the zero product. Let $\mathfrak{A}=A \oplus E$ and $\mathfrak{B}=A \oplus \mathfrak{A}$ be constructed as in Proposition 3.3.1. Define the action of $A$ on $\mathfrak{A}$ to be

$$
a \cdot(b, y)=(b, y) \cdot a=(b, y) \square(a, 0) \quad \forall a \in A,(b, y) \in \mathfrak{A} .
$$

If $\mathfrak{A}$ is left strongly Arens irregular then $\mathfrak{B}$ is strongly Arens irregular that admits a subalgebra that is Arens regular and not strongly Arens regular.

Proof We first need to show that the actions defined above are indeed actions. So, let

$f, g \in A,(b, y) \in \mathfrak{A}$ then

$$
\begin{aligned}
{[f g] \cdot(b, y) } & =(b, y) \square(f g, 0) \\
& =(b, y) \square(g f, 0) \\
& =(b, y) \square[(g, 0) \square(f, 0)] \\
& =[(b, y) \square(g, 0)] \square(f, 0) \\
& =f \cdot[(b, y) \square(g, 0)] \\
& =f \cdot[g \cdot(b, y)] .
\end{aligned}
$$


On the other hand,

$$
\begin{aligned}
(b, y) \cdot[f g] & =(b, y) \square(f g, 0) \\
& =(b, y) \square[(f, 0) \square(g, 0)] \\
& =[(b, y) \square(f, 0)] \square(g, 0) \\
& =[(b, y) \square(f, 0)] \cdot g \\
& =[(b, y) \cdot f] \cdot g .
\end{aligned}
$$

So $\mathfrak{B}$ is indeed a Banach algebra. To show that $\mathfrak{B}$ is strongly Arens irregular, we first look at $Z_{t}^{1}\left(\mathfrak{A}^{* *}\right)$ and show that the map $A^{* *} \ni \Psi \mapsto \Lambda \odot \Psi \in \mathfrak{A}^{* *}$ being $w^{*}-w^{*}$ continuous is equivalent to $\Lambda \in Z_{t}^{1}\left(\mathfrak{A}^{* *}\right)$. So, let $\left(\Lambda_{A}, \Lambda_{E}\right) \in \mathfrak{A}^{* *}$ and take $\left(\mu_{\alpha}, \nu_{\alpha}\right)$ a net in $\mathfrak{A}^{* *}$ converging $w^{*}$ to 0 . Then as

$$
\left(\Lambda_{A}, \Lambda_{E}\right) \odot\left(\mu_{\alpha}, \nu_{\alpha}\right)=\left(\Lambda_{A} \odot \mu_{\alpha}, \Lambda_{A} \odot \nu_{\alpha}+\Lambda_{E} \odot \mu_{\alpha}\right)
$$

by Lemma 4.1.1, we have $\left(\Lambda_{A}, \Lambda_{E}\right) \in Z_{t}^{1}\left(\mathfrak{A}^{* *}\right)$ if and only if $\Lambda_{A} \odot \mu_{\alpha}, \Lambda_{A} \odot \nu_{\alpha}$, and $\Lambda_{E} \odot \mu_{\alpha}$ converge $w^{*}$ to 0 . Since both $A$ and $\mathfrak{A}$ are LSAI, we have $\left(\Lambda_{A}, \Lambda_{E}\right) \in Z_{t}^{1}\left(\mathfrak{A}^{* *}\right)=\mathfrak{A}$ if and only if $\Lambda_{A} \odot \mu_{\alpha}$ and $\Lambda_{E} \odot \mu_{\alpha}$ converge $w^{*}$ to 0 .

Now let $(\Phi, \Lambda) \in Z_{t}^{1}\left(\mathfrak{B}^{* *}\right)$. By Lemma 4.1.1 we have that $\Phi \in Z_{t}^{1}\left(A^{* *}\right)=A$ and the map $A^{* *} \ni \Psi \mapsto \Lambda \odot \Psi$ is $w^{*}-w^{*}$ continuous. Thus $\Lambda \in Z_{t}^{1}\left(\mathfrak{A}^{* *}\right)=\mathfrak{A}$ which gives us that $\mathfrak{B}$ is LSAI.

Now let $(\Phi, \Lambda) \in Z_{t}^{2}\left(\mathfrak{B}^{* *}\right)$ and take a net $\left(\mu_{\alpha}, \nu_{\alpha}\right)$ in $\mathfrak{B}^{* *}$ that converges $w^{*}$ to 0 . Then $\left(\mu_{\alpha}, \nu_{\alpha}\right) \diamond(\Phi, \Lambda)$ converges $w^{*}$ to 0 . Thus $\left(\mu_{\alpha} \diamond \Phi, \Lambda \odot \mu_{\alpha}+\nu_{\alpha} \diamond \Phi\right)$ converges $w^{*}$ to 0 and so each of $\mu_{\alpha} \diamond \Phi, \Lambda \odot \mu_{\alpha}$, and $\nu_{\alpha} \diamond \Phi$ converges $w^{*}$ to 0 . This implies that $\Phi \in Z_{t}^{2}\left(A^{* *}\right)=A$ and we have already shown that $\Lambda \odot \mu_{\alpha}$ converging $w^{*}$ to 0 implies that $\Lambda \in \mathfrak{A}$. Thus $\mathfrak{B}$ is strongly Arens irregular.

Since $E$ is a Banach space endowed with the zero product, then $Z_{t}\left(E^{* *}\right)=E^{* *}$ and so is Arens regular. Since $\left|E^{* *}\right|>|E|$ then $E$ is not strongly Arens irregular. It is not obvious that $E$ is in fact a subalgebra of $\mathfrak{B}$. If we identify $E$ with $0 \oplus E$ then the following simple calculation shows that it is. Let $(0, f),(0, g) \in 0 \oplus E$ then 
$(0,(0, f))$ and $(0,(0, g)) \in \mathfrak{B}$ and we have

$$
\begin{aligned}
(0,(0, f)) \square(0,(0, g)) & =(0,0 \cdot(0, g)+(0, f) \cdot 0) \\
& =(0,(0, g) \square(0,0)+(0, f) \square(0,0)) \\
& =(0,0) .
\end{aligned}
$$

We give two examples of algebras $A$ and $E$. The first is a specific example which prompted the idea to form the algebra in an abstract manner.

\section{Example 1:}

Let $A=\ell_{1}(G)$ and $E=\left(M_{\ell_{\infty}(G)}\right)_{\perp}$ where $G$ is an infinite commutative discrete group. The product for each of these spaces is the convolution type product introduced earlier in $\left(\mathcal{N}\left(\ell_{p}(G)\right)\right)=\ell_{1}(G) \oplus\left(M_{\ell_{\infty}(G)}\right)_{\perp}$. With this we see that $A$ is strongly Arens irregular and that $E$ is an infinite dimensional Banach algebra that is Arens regular and not SAI. We have seen already that $A \oplus E$ is left (but not right) strongly Arens irregular. Applying the above theorem we see that $\ell_{1}(G) \oplus \mathcal{N}\left(\ell_{p}(G)\right)$ is strongly Arens irregular.

\section{Example 2:}

Let $A=L_{1}(G), G$ any infinite locally compact commutative group. Take the product on $A$ to be the usual convolution product and thus $A$ is strongly Arens irregular. Let $E=A$ as Banach spaces and endow $E$ with the trivial product. Denote this algebra by $L_{1}(G)_{0}$ to distinguish it from the usual convolution algebra $L_{1}(G)$. Thus $E$ is an infinite dimensional Banach algebra that is Arens regular. Define the action of $A$ on $E$ from the right to be $a \cdot x=a * x$. To show that the map $\Psi \mapsto \Lambda \cdot \Psi$ is continuous from $A^{* *}$ to $E^{* *}$ if and only if $\Lambda \in E$, let $\Lambda \in E^{* *}$ and take a net $\mu_{\alpha}$ in $A^{* *}$ that converges $w^{*}$ to 0 . Then $\Lambda \cdot \mu_{\alpha}=\Lambda \odot \mu_{\alpha}$ which forces $\Lambda \in Z_{t}^{1}\left(A^{* *}\right)=A=E$. Thus we have again satisfied the conditions of the above theorem and so the Banach algebra $\mathrm{E}_{1}(G) \oplus\left[L_{1}(G) \oplus L_{1}(G)_{0}\right]$ is strongly Arens irregular with an infinite dimensional subalgebra that is Arens regular and not SAI. 
One last remark on this idea is to point out that in both examples given, because of the product defined on $A \oplus E$, the algebra $E$ must have the zero product. Any other product on $E$ would not yield a subalgebra of $(A \oplus E, \square)$. It is unknown whether there exists an example where $A$ is a Banach algebra that is SAI which admits a subalgebra that is AR and not SAI where the product restricted to the subalgebra is not the trivial one.

We would also like to point out that the conditions in Lemma 4.1.1 are all necessary. In other words, we may ask the question as to whether there is a relation between the topological centre of $A^{* *}$ and the projection onto the first component, $\pi_{A^{* *}}: A^{* *} \oplus E^{* *} \rightarrow A^{* *}$, of the topological centre of $A^{* *} \oplus E^{* *}$. The answer is that in general the only relation is the trivial inclusion of $\pi_{A^{* *}}\left(Z_{t}^{1,2}\left(A^{* *} \ominus E^{* *}\right)\right) \subset Z_{t}^{1,2}\left(A^{* *}\right)$. A simple example is similar to example 2, taking $G$ to be any infinite locally compact commutative group. If we set $A=L_{1}(G)_{0}$ and $E=L_{1}(G)$ then define the action of $A$ on $E$ to be the product in $A$. With this setup we can easily see that $Z_{t}^{1,2}\left(A^{* *}\right)=A^{* *}$ but from Lemma 4.1 .1 we know that if $(\Phi, \Lambda) \in Z_{t}^{1}\left(A^{* *} \oplus E^{* *}\right)$ then the map $M \mapsto \Phi \cdot M=\Phi \odot M$ is $w^{*}-w^{*}$ continuous which imposes the condition that $\Phi \in Z_{t}^{1}\left(L_{1}(G)^{* *}\right)=L_{1}(G)$ and similarly for the second topological centre. Thus we have $\pi_{A^{* *}}\left(Z_{t}^{1,2}\left(A^{* *} \oplus E^{* *}\right)\right)=A \neq A^{* *}$. 


\section{Chapter 5}

\section{Jordan Topological Centres}

In this section we use $\mathcal{N}\left(\ell_{p}(G)\right)$ as motivation to investigate what happens to the topological centre of a Banach algebra that is highly non-commutative when we force commutivity. This forcing of commutivity is done by looking at the Jordan algebra of the original Banach algebra. We first start by introducing the Jordan product and defining the notion of a Jordan topological centre. We then give some general results for any Banach algebra. Finally we determine the Jordan centre for $\ell_{1}(G)$ and $\mathcal{N}\left(\ell_{p}(G)\right)$ for a few classes of groups by developing a strengthened version of the factorization technique used in section 3.

\subsection{General results for Banach algebras}

We start by defining the Jordan product for a given algebra.

Definition 5.1.1 Let $(A, *)$ be an algebra. We denote by $*_{J}$ the Jordan product and it is defined by

$$
a *_{J} b=\frac{a * b+b * a}{2} \quad \forall a, b \in A
$$

The Jordan product is a bilinear form that symmetrizes a noncommutative algebra. It is easy to see that this product is not necessarily associative, for if $a, b, c \in A$ 
then

$$
\begin{aligned}
\left(a *_{J} b\right) *_{J} c & =\left(\frac{a * b+b * a}{2}\right) *_{J} c \\
& =\frac{\left(\frac{a * b+b * a}{2}\right) * c+c *\left(\frac{a * b+b * a}{2}\right)}{2} \\
& =\frac{(a * b) * c+(b * a) * c+c *(a * b)+c *(b * a)}{4}
\end{aligned}
$$

and

$$
\begin{aligned}
a *_{J}\left(b *_{J} c\right) & =a *_{J}\left(\frac{b * c+c * b}{2}\right) \\
& =\frac{a *\left(\frac{b * c+c * b}{2}\right)+\left(\frac{b * c+c * b}{2}\right) * a}{2} \\
& =\frac{a *(b * c)+a *(c * b)+(b * c) * a+(c * b) * a}{4} \\
& =\frac{(a * b) * c+a *(c * b)+(b * c) * a+c *(b * a)}{4} .
\end{aligned}
$$

Now,

$$
\begin{aligned}
& \left(a *_{J} b\right) *_{J} c-a *_{J}\left(b *_{J} c\right) \quad=0 \\
& \Leftrightarrow(b * a) * c+c *(a * b)-a *(c * b)-(b * c) * a=0 \\
& \Leftrightarrow b *(a * c)+(c * a) * b-(a * c) * b-b *(c * a)=0 \\
& \Leftrightarrow \quad b *((a * c)-(c * a))-((a * c)-(c * a)) * b=0
\end{aligned}
$$

Taking $A=\mathcal{N}\left(\ell_{p}(G)\right)=\ell_{1}(G) \oplus\left(M_{\ell_{\infty}(G)}\right)_{\perp}$ and $a, c \in \ell_{1}(G), \quad b \in\left(M_{\ell_{\infty}(G)}\right)_{\perp}$ we see that this product may not be associative. It is clear however, that if $A$ is commutative then the Jordan product is the same is the original product on $A$. Fortunately the Arens extensions of a product do not require associativity. The next proposition shows that the Arens extensions of the Jordan product give the expected commutivity in the bidual level. 
Proposition 5.1.1 Let $(A, *)$ be a Banach algebra. Then the first and second Arens extensions of the Jordan product, denoted $\odot_{J}$ and $\diamond_{J}$ respectively, are

$$
\Phi \odot_{J} \Lambda=\frac{\Phi \odot \Lambda+\Lambda \diamond \Phi}{2}=\Lambda \diamond_{J} \Phi \quad \forall \Phi, \Lambda \in A^{* *}
$$

Proof Fix $\phi_{0} \in A$ and let $\Lambda \in A^{* *}$. Take a net $\lambda_{\alpha}$ in $A$ such that $\Lambda=w^{*}-\lim _{\alpha} \lambda_{\alpha}$. Then

$$
\begin{aligned}
\phi_{0} \odot_{J} \Lambda & =w^{*}-\lim _{\alpha} \phi_{0} \odot_{J} \lambda_{\alpha} \\
& =w^{*}-\lim _{\alpha} \phi_{0} *_{J} \lambda_{\alpha} \\
& =w^{*}-\lim _{\alpha} \frac{1}{2}\left(\phi_{0} * \lambda_{\alpha}+\lambda_{\alpha} * \phi_{0}\right) \\
& =w^{*}-\lim _{\alpha} \frac{1}{2}\left(\phi_{0} \odot \lambda_{\alpha}+\lambda_{\alpha} \diamond \phi_{0}\right) \\
& =\frac{1}{2}\left(\phi_{0} \odot \Lambda+\Lambda \diamond \phi_{0}\right) .
\end{aligned}
$$

Now let $\Phi \in A^{* *}$ and approximate $\Phi$ by a net $\phi_{\alpha}$ in $A$. Then using the above calculation

$$
\begin{aligned}
\Phi \odot_{J} \Lambda & =w^{*}-\lim _{\alpha} \phi_{\alpha} \odot_{J} \Lambda \\
& =w^{*}-\lim _{\alpha} \frac{1}{2}\left(\phi_{\alpha} \odot \Lambda+\Lambda \diamond \phi_{\alpha}\right) \\
& =\frac{1}{2}(\Phi \odot \Lambda+\Lambda \diamond \Phi) .
\end{aligned}
$$

To show that $\Phi \odot_{J} \Lambda=\Lambda \diamond_{J} \Phi$ fix $\phi_{0} \in A$ and let $\Lambda \in A^{* *}$. Take a net $\lambda_{\alpha}$ in $A$ such that $\Lambda=w^{*}-\lim \lambda_{\alpha}$. Then

$$
\begin{aligned}
\Lambda \diamond_{J} \phi_{0} & =w^{*}-\lim _{\alpha} \lambda_{\alpha} \diamond_{J} \phi_{0} \\
& =w^{*}-\lim _{\alpha} \lambda_{\alpha} *_{J} \phi_{0} \\
& =w^{*}-\lim _{\alpha} \frac{1}{2}\left(\lambda_{\alpha} * \phi_{0}+\phi_{0} * \lambda_{\alpha}\right) \\
& =w^{*}-\lim _{\alpha} \frac{1}{2}\left(\lambda_{\alpha} \diamond \phi_{0}+\phi_{0} \odot \lambda_{\alpha}\right) \\
& =\frac{1}{2}\left(\Lambda \diamond \phi_{0}+\phi_{0} \odot \Lambda\right) .
\end{aligned}
$$


Now let $\phi_{\alpha}$ be a net in $A$ such that $\Phi=w^{*}-\lim _{\alpha} \phi_{\alpha}$. Then

$$
\begin{aligned}
\Lambda \diamond_{J} \Phi & =w^{*}-\lim _{\alpha} \Lambda \diamond_{J} \phi_{\alpha} \\
& =w^{*}-\lim _{\alpha} \frac{1}{2}\left(\Lambda \diamond \phi_{\alpha}+\phi_{\alpha} \odot \Lambda\right) \\
& =\frac{1}{2}(\Lambda \diamond \Phi+\Phi \odot \Lambda) \\
& =\Phi \odot_{J} \Lambda
\end{aligned}
$$

Remark: For any algebra $(A, *)$ we will denote the Jordan algebra $\left(A, *_{J}\right)$ simply by $A_{J}$. The Jordan product produces a commutative (possibly non-associative) algebra and thus in light of the previous proposition we have that $Z_{t}^{1}\left(A_{J}^{* *}\right)=Z_{t}^{2}\left(A_{J}^{* *}\right)$. Also from the previous proposition it is easy to see that $Z_{t}^{1}\left(A_{J}^{* *}\right)$ consists of all the elements of the form $\Phi \in A^{* *}$ such that the map $n \mapsto \Phi \odot n+n \diamond \Phi$ is $w^{*}-w^{*}$ continuous.

Definition 5.1.2 Let $A$ be a Banach algebra. The Jordan topological centre of $A^{* *}$ is the set

$$
Z_{t}\left(A_{J}^{* *}\right)=\left\{m \in A^{* *} \mid \text { the map } n \mapsto m \odot_{J} n \text { is } w^{*}-w^{*} \text { continuous }\right\}
$$

The next proposition shows how the toplogical centre of the bidual of the Jordan algebra relates to the first and second centres of the bidual of the original algebra.

Proposition 5.1.2 Let $A$ be a Banach algebra. Then

$$
Z_{t}^{1}\left(A^{* *}\right) \cap Z_{t}^{2}\left(A^{* *}\right)=Z_{t}\left(A_{J}^{* *}\right) \cap\left(Z_{t}^{1}\left(A^{* *}\right) \cup Z_{t}^{2}\left(A^{* *}\right)\right)
$$

Proof Clearly $Z_{t}^{1}\left(A^{* *}\right) \cap Z_{t}^{2}\left(A^{* *}\right) \subset\left(Z_{t}^{1}\left(A^{* *}\right) \cup Z_{t}^{2}\left(A^{* *}\right)\right)$. To show that $Z_{t}^{1}\left(A^{* *}\right) \cap Z_{t}^{2}\left(A^{* *}\right) \subset Z_{t}\left(A_{J}^{* *}\right)$ let $\Phi \in Z_{t}^{1}\left(A^{* *}\right) \cap Z_{t}^{2}\left(A^{* *}\right)$ and let $\mu_{\alpha}$ be a net in $A^{* *}$ converging $w^{*}$ to zero. Then

$$
\Phi \odot_{J} \mu_{\alpha}=\frac{1}{2}\left(\Phi \odot \mu_{\alpha}+\mu_{\alpha} \diamond \Phi\right) \stackrel{\alpha}{\longrightarrow} 0
$$


since both terms converge to zero $\left(w^{*}\right)$ which gives the desired inclusion.

To show that $Z_{t}\left(A_{J}^{* *}\right) \cap\left(Z_{t}^{1}\left(A^{* *}\right) \cup Z_{t}^{2}\left(A^{* *}\right)\right) \subset Z_{t}^{1}\left(A^{* *}\right) \cap Z_{t}^{2}\left(A^{* *}\right)$ assume the contrary that there exists $\Lambda \in Z_{t}\left(A_{J}^{* *}\right) \cap\left(Z_{t}^{1}\left(A^{* *}\right) \cup Z_{t}^{2}\left(A^{* *}\right)\right)$ such that $\Lambda \notin Z_{t}^{1}\left(A^{* *}\right) \cap Z_{t}^{2}\left(A^{* *}\right)$. This implies that $\Lambda$ is either in $Z_{t}^{2}\left(A^{* *}\right)$ or $Z_{t}^{1}\left(A^{* *}\right)$ but not

both. Without loss of generality assume that $\Lambda \in Z_{t}^{2}\left(A^{* *}\right)$. As $\Lambda \notin Z_{t}^{1}\left(A^{* *}\right)$ there exists a net $\mu_{\alpha}$ converging $w^{*}$ to zero such that $\Lambda \odot \mu_{\alpha}$ does not converge to zero $\left(w^{*}\right)$. Since $\Lambda \in Z_{t}\left(A_{J}^{* *}\right)$ we have that $\Lambda \odot \mu_{\alpha}+\mu_{\alpha} \diamond \Lambda$ converges to zero. Therefore $\lim _{\alpha} \Lambda \odot \mu_{\alpha}=-\lim _{\alpha} \mu_{\alpha} \diamond \Lambda\left(w^{*}\right)$ but because $\Lambda \in Z_{t}^{2}\left(A^{* *}\right)$ the right side of the equality is zero, a contradiction.

Definition 5.1.3 Let $A$ be a Banach algebra. We say that $A$ is Jordan strongly Arens irregular (JSAI) if $Z_{t}\left(A_{J}^{* *}\right)=A$ and $A$ is Jordan Arens regular (JAR) if $Z_{t}\left(A_{J}^{* *}\right)=A^{* *}$.

\subsection{Jordan topological centre of $\ell_{1}(G)$}

The techniques that we will use to determine the Jordan centre of $\ell_{1}(G)$ are very similar to those that we used when calculating the first and second centre of $\ell_{1}(G)$. We state now the definition of the kind of factorization that will be needed and then prove a theorem very much like Theorem 2.2.1.

Definition 5.2.1 Let $A$ be a Banach algebra and let $\kappa$ be a cardinal number. We say that $A^{*}$ has the simultaneous $A^{* *}$-factorization of level $\kappa$ if for every net $\left(f_{\alpha}\right)_{\alpha \in I}$ with $|I| \leq \kappa$ there exists a net $\left(\psi_{\alpha}\right)_{\alpha \in I} \subset \operatorname{Ball}\left(A^{* *}\right)$ and a single function $f \in A^{*}$ such that the factorizations

$$
f_{\alpha}=\psi_{\alpha} \odot f \quad \text { and } \quad f_{\alpha}=f \diamond \psi_{\alpha}
$$

hold for every $\alpha \in I$.

We would just like to point out that $\ell_{1}(G)^{*}$ has both the left $\ell_{1}(G)^{* *}$ and right $\ell_{1}(G)^{* *}$-factorization properties of level $\kappa(G)$. This does not imply a priori that $\ell_{1}(G)^{*}$ has the simultaneous $\ell_{1}(G)^{* *}$-factorization property of level $\kappa(G)$ because a 
sequence $\left(f_{\alpha}\right)_{\alpha \in I} \subset \ell_{1}(G)^{*}$ factorizes in the following way, $f_{\alpha}=\psi_{\alpha} \odot f=g \diamond \psi_{\alpha}$, and generally the functionals $f$ and $g$ are not equal.

Theorem 5.2.1 Let $A$ be a Banach algebra with the Mazur property of level $\kappa \geq \aleph_{0}$. If $A^{*}$ has the simultaneous $A^{* *}$-factorization property of level $\kappa$ then $A$ is JSAI.

Proof Let $m \in Z_{t}\left(A_{J}^{* *}\right)$ and take a net $\left(h_{\alpha}\right)_{\alpha \in I} \subset \operatorname{Ball}\left(A^{*}\right)$ with $|I| \leq \kappa$ which converges $w^{*}$ to 0 . Since $A$ has the Mazur property of level $\kappa$, we only need to show that $\left\langle m, h_{\alpha}\right\rangle$ converges to 0 . It is sufficient to show that every convergent subnet $\left(\left\langle m, h_{\alpha}\right\rangle\right)_{\alpha \in I}$ converges to 0 . Let $\left(\left\langle m, h_{\alpha_{\beta}}\right\rangle\right)_{\beta}$ be a convergent subnet. As $A^{*}$ has the simultaneous $A^{* *}$-factorization property of level $\kappa$ we have that $h_{\alpha}=$ $\psi_{\alpha} \odot h=h \diamond \psi_{\alpha}=\psi_{\alpha} \odot_{J} h$ where $\left(\psi_{\alpha}\right)_{\alpha \in I} \subset \operatorname{Ball}\left(A^{* *}\right)$ and $h \in A^{*}$. Since the net $\left(\psi_{\alpha_{\beta}}\right)_{\beta} \subset$ Ball $\left(A^{* *}\right)$, by Alaoglu's Theorem, there exists a $w^{*}$-convergent subnet $\left(\psi_{\alpha_{\beta_{\gamma}}}\right)_{\gamma}$. Let $E=w^{*}-\lim _{\gamma} \psi_{\alpha_{\beta_{\gamma}}} \in \operatorname{Ball}\left(A^{* *}\right)$. Now, using the fact that $h_{\alpha}$ converges $w^{*}$ to 0 we have for all $a \in A$

$$
\begin{aligned}
\left\langle E \odot_{J} h, a\right\rangle & =\left\langle E, h \odot_{J} a\right\rangle=\lim _{\gamma}\left\langle\psi_{\alpha_{\beta_{\gamma}}}, h \odot_{J} a\right\rangle \\
& =\lim _{\gamma}\left\langle\psi_{\alpha_{\beta_{\gamma}}} \odot_{J} h, a\right\rangle=\lim _{\gamma}\left\langle h_{\alpha_{\beta_{\gamma}}}, a\right\rangle=0 .
\end{aligned}
$$

This implies that $E \odot_{J} h=0$. So, as $m \in Z_{t}^{1}\left(A^{* *}\right)$;

$$
\begin{aligned}
\lim _{\beta}\left\langle m, h_{\alpha_{\beta}}\right\rangle & =\lim _{\gamma}\left\langle m, h_{\alpha_{\beta_{\gamma}}}\right\rangle \\
& =\lim _{\gamma}\left\langle m, \psi_{\alpha_{\beta_{\gamma}}} \odot_{J} h\right\rangle \\
& =\lim _{\gamma}\left\langle m \odot_{J} \psi_{\alpha_{\beta_{\gamma}}}, h\right\rangle \\
& =\left\langle m \odot_{J} E, h\right\rangle \\
& =\left\langle m, E \odot_{J} h\right\rangle \\
& =0 .
\end{aligned}
$$

Hence, $m \in A$, and $A$ is JSAI.

Now that we have an analogous result to Theorem 2.2.1 we just need to show that $\ell_{1}(G)^{*}$ enjoys the simultaneous $\ell_{1}(G)^{* *}$-factorization property of level $\kappa(G)$. The 
next theorem show that we can get this simultaneous factorization for any countable ICC group. $G$ being an ICC group means that any element $g \in G$ with $g \neq e$, belongs to an infinite conjugacy class. A large class of examples of countable ICC groups are the free groups on $n$ generators, where $2 \leq n \leq \aleph_{0}$.

Theorem 5.2.2 Let $G$ be a countable ICC Group. Then there exists a sequence $\left(\psi_{n}\right)_{n \in \mathbb{N}} \subset{\overline{\delta_{G}}}^{w^{*}} \subset \operatorname{Ball}\left(\ell_{\infty}(G)^{*}\right)$ such that for every sequence $\left(f_{n}\right)_{n \in \mathbb{N}} \subset \operatorname{Ball}\left(\ell_{\infty}(G)\right)$ there is a single function $f \in \operatorname{Ball}\left(\ell_{\infty}(G)\right)$ such that the factorizations

$$
f_{n}=\psi_{n} \odot f=f \diamond \psi_{n}=\psi_{n} \odot \jmath f
$$

hold for all $n \in \mathbb{N}$.

Proof Let $\left\{A_{n}\right\}_{n \in \mathbb{N}}$ be a covering of $G$ by finite sets such that $\forall n \leq m \in \mathbb{N}$ we have $e \in A_{n} \subset A_{m}$, where $e$ is the identity element in $G$.

Now consider the set $\tilde{I}=\mathbb{N} \times \mathbb{N}$. For $\tilde{n}=(n, h) \in \tilde{I}$ we set $A_{\tilde{n}}:=A_{n}$. We construct a net $\left(y_{\tilde{n}}\right)_{\tilde{n} \in \tilde{I}}$ with the following properties:

$$
\begin{array}{lll}
\text { i) } & A_{\tilde{n}} y_{\tilde{n}} \cap A_{\tilde{m}} y_{\tilde{m}}=\varnothing & \text { if } \tilde{n} \neq \tilde{m} \\
\text { ii) } & y_{\tilde{n}} A_{\tilde{n}} \cap y_{\tilde{m}} A_{\tilde{m}}=\varnothing & \text { if } \tilde{n} \neq \tilde{m} \\
\text { iii) } & y_{\tilde{n}} A_{\tilde{n}} \cap A_{\tilde{m}} y_{\tilde{m}}=\varnothing & \text { if } \tilde{n} \neq \tilde{m} \\
\text { iv) } & y_{\tilde{n}} \cap A_{\tilde{m}} y_{\tilde{m}}=\varnothing & \text { if } \tilde{n} \neq \tilde{m} \\
\text { v) } & y_{\tilde{n}} \cap y_{\tilde{m}} A_{\tilde{m}}=\varnothing & \text { if } \tilde{n} \neq \tilde{m} \\
\text { vi) } & y_{\tilde{n}} A_{\tilde{n}} \cap A_{\tilde{n}} y_{\tilde{n}}=\left\{y_{\tilde{n}}\right\} & \forall \tilde{n} \in \tilde{I}
\end{array}
$$

For this we well order $\tilde{I}$ by $\leq$ with the property that for each $\tilde{n} \in \tilde{I}$ the set $\{\tilde{m} \in \tilde{I} \mid \tilde{m} \leq \tilde{n}\}$ is finite. Fix $\tilde{n} \in \tilde{I}$. We construct the desired net by induction by assuming that we have found $y_{\tilde{m}}$ satisfying the above six properties $\forall \tilde{m}<\tilde{n}$. For each $\tilde{m}<\tilde{n}$ set

$$
K_{\tilde{m}}=A_{\tilde{n}}^{-1} A_{\tilde{m}} y_{\tilde{m}} \cup y_{\tilde{m}} A_{\tilde{m}} A_{\tilde{n}}^{-1} \cup A_{\tilde{m}} y_{\tilde{m}} A_{\tilde{n}}^{-1} \cup A_{\tilde{m}} y_{\tilde{m}} \cup y_{\tilde{m}} A_{\tilde{m}}
$$

Enumerate $A_{\tilde{n}}$ as $A_{\tilde{n}}=\left\{1, g_{1}, g_{2}, \ldots, g_{k}\right\}$. As $G$ is ICC we have that $\left[g_{i}\right]$ is infinite for each $1 \leq i \leq k$ (where $\left[g_{i}\right]$ is the conjugacy class of $g_{i}$ ). Since $A_{\tilde{n}}$ is finite there exist 
infinitely many $h \in G$ such that $h g_{i} h^{-1} \notin A_{\tilde{n}}$ for every $1 \leq i \leq k$. Choose one of these elements $h \in G$ such that $h \notin K_{\tilde{m}} \forall \tilde{m}<\tilde{n}$. This is possible since $K_{\tilde{m}}$ is a finite union of finite sets. Set $y_{\tilde{n}}=h$, then $y_{\tilde{n}}$ satisfies the 6 properties listed above.

We define a relation on $\tilde{I}$ by

$$
\tilde{n} \prec \tilde{m} \Longleftrightarrow n \leq m \text {. }
$$

Clearly, this relation is transitive and directs $\tilde{I}$. Note that whenever $\tilde{n} \prec \tilde{m}$ we have that $\chi_{A_{\tilde{n}}} \chi_{A_{\tilde{m}}}=\chi_{A_{\tilde{n}}}$.

For $\tilde{n}=(n, h) \in \tilde{I}$ let $u_{\tilde{n}}=u_{(n, h)}:=\chi_{A_{(n, h)}}$ and consider the functions

$$
v_{\tilde{n}}=v_{(n, h)}:=r_{y_{(n, h)}^{-1}}\left(u_{(n, h)} f_{h}\right)
$$

and

$$
w_{\tilde{n}}=w_{(n, h)}:=l_{y_{(n, h)}^{-1}}\left(u_{(n, h)} f_{h}\right)
$$

For $\tilde{n}=(n, h), \tilde{m}=(m, k) \in I$ with $\tilde{n} \neq \tilde{m}$ by (5.1) we have the following results:

$$
\begin{aligned}
\operatorname{supp}\left(v_{\tilde{n}}\right) \cap \operatorname{supp}\left(v_{\tilde{m}}\right) & =\operatorname{supp}\left(u_{(n, h)} f_{h}\right) y_{(n, h)} \cap \operatorname{supp}\left(u_{(m, k)} f_{k}\right) y_{(m, k)} \\
& \subset \operatorname{supp}\left(u_{(n, h)}\right) y_{(n, h)} \cap \operatorname{supp}\left(u_{(m, k)}\right) y_{(m, k)} \\
& =A_{(n, h)} y_{(n, h)} \cap A_{(m, k)} y_{(m, k)} \\
& =A_{\tilde{n}} y_{\tilde{n}} \cap A_{\tilde{m}} y_{\tilde{m}} \\
& =\varnothing
\end{aligned}
$$

and

$$
\begin{aligned}
\operatorname{supp}\left(w_{\tilde{n}}\right) \cap \operatorname{supp}\left(w_{\tilde{m}}\right) & =y_{(n, h)} \operatorname{supp}\left(u_{(n, h)} f_{h}\right) \cap y_{(m, k)} \operatorname{supp}\left(u_{(m, k)} f_{k}\right) \\
& \subset y_{(n, h)} \operatorname{supp}\left(u_{(n, h)}\right) \cap y_{(m, k)} \operatorname{supp}\left(u_{(m, k)}\right) \\
& =y_{(n, h)} A_{(n, h)} \cap y_{(m, k)} A_{(m, k)} \\
& =y_{\tilde{n}} A_{\tilde{n}} \cap y_{\tilde{m}} A_{\tilde{m}} \\
& =\varnothing
\end{aligned}
$$


and

$$
\begin{aligned}
\operatorname{supp}\left(v_{\tilde{n}}\right) \cap \operatorname{supp}\left(w_{\tilde{m}}\right) & =\operatorname{supp}\left(u_{(n, h)} f_{h}\right) y_{(n, h)} \cap y_{(m, k)} \operatorname{supp}\left(u_{(m, k)} f_{k}\right) \\
& \subset \operatorname{supp}\left(u_{(n, h)}\right) y_{(n, h)} \cap y_{(m, k)} \operatorname{supp}\left(u_{(m, k)}\right) \\
& =A_{(n, h)} y_{(n, h)} \cap y_{(m, k)} A_{(m, k)} \\
& =A_{\tilde{n}} y_{\tilde{n}} \cap y_{\tilde{m}} A_{\tilde{m}} \\
& =\varnothing
\end{aligned}
$$

and for each $\tilde{n} \in \tilde{I}$ we have

$$
\begin{aligned}
\operatorname{supp}\left(w_{\tilde{n}}\right) \cap \operatorname{supp}\left(v_{\tilde{n}}\right) & =y_{(n, h)} \operatorname{supp}\left(u_{(n, h)} f_{h}\right) \cap \operatorname{supp}\left(u_{(n, h)} f_{h}\right) y_{(n, h)} \\
& \subset y_{(n, h)} \operatorname{supp}\left(u_{(n, h)}\right) \cap \operatorname{supp}\left(u_{(n, h)}\right) y_{(n, h)} \\
& =y_{(n, h)} A_{(n, h)} \cap A_{(n, h)} y_{(n, h)} \\
& =y_{\tilde{n}} A_{\tilde{n}} \cap A_{\tilde{n}} y_{\tilde{n}} \\
& =\left\{y_{\tilde{n}}\right\} .
\end{aligned}
$$

Now for each $h \in \mathbb{N}$ we set $g_{h}(x)=f_{h}(e) \forall x \in G$, and then we define $f$ pointwise by

$$
f:=\sum_{(n, h) \in \tilde{I}}\left[r_{y_{(n, h)}^{-1}}\left(u_{(n, h)} f_{h}\right)+l_{y_{(n, h)}^{-1}}\left(u_{(n, h)} f_{h}\right)-\chi_{y_{(n, h)}} g_{h}\right] .
$$

We claim that $f \in \operatorname{Ball}\left(\ell_{\infty}(G)\right)$. Indeed, let $x \in G$. From the above relations we have that $\operatorname{supp}\left(\chi_{y_{(n, h)}} g_{h}\right)=\left\{y_{(n, h)}\right\}$ and $\operatorname{supp}\left(w_{(n, h)}\right) \cap \operatorname{supp}\left(v_{(n, h)}\right)=\left\{y_{(n, h)}\right\}$. Case $1:\left(x=y_{(n, h)}\right.$ for some $\left.(n, h) \in \tilde{I}\right)$ 
Then

$$
\begin{aligned}
f(x) & =\sum_{(n, h) \in \tilde{I}}\left[r_{y_{(n, h)}^{-1}}\left(u_{(n, h)} f_{h}\right)+l_{y_{(n, h)}^{-1}}\left(u_{(n, h)} f_{h}\right)-\chi_{y_{(n, h)}} g_{h}\right](x) \\
& =r_{y_{(n, h)}^{-1}}\left(u_{(n, h)} f_{h}\right)\left(y_{(n, h)}\right)+l_{y_{(n, h)}^{-1}}\left(u_{(n, h)} f_{h}\right)\left(y_{(n, h)}\right)-\chi_{y_{(n, h)}} g_{h}\left(y_{(n, h)}\right) \\
& =\left(u_{(n, h)} f_{h}\right)(e)+\left(u_{(n, h)} f_{h}\right)(e)-g_{h}(e) \\
& =f_{h}(e)+f_{h}(e)-g_{h}(e) \\
& =2 f_{h}(e)-f_{h}(e) \\
& =f_{h}(e) .
\end{aligned}
$$

Thus, $\left|f\left(y_{(n, h)}\right)\right| \leq 1$ for all $(n, h) \in \tilde{I}$.

Case 2: $\left(x \neq y_{(n, h)} \forall(n, h) \in \tilde{I}\right)$

Case 2a: $\left(x \in \operatorname{supp}\left(w_{\tilde{n}}\right)\right.$ for some $\left.\tilde{n} \in \tilde{I}\right)$

Then $|f(x)|=\left|w_{(n, h)}\right| \leq\left|f_{h}(x)\right| \leq 1$.

Case $2 \mathrm{~b}:\left(x \in \operatorname{supp}\left(v_{\tilde{n}}\right)\right.$ for some $\left.\tilde{n} \in \tilde{I}\right)$

Then $|f(x)|=\left|v_{(n, h)}\right| \leq\left|f_{h}(x)\right| \leq 1$.

Case 2c: $\left(x \notin \operatorname{supp}\left(w_{\tilde{n}}\right) \cup \operatorname{supp}\left(v_{\tilde{n}}\right)\right)$

Then $f(x)=0$.

Hence, $f \in \operatorname{Ball}\left(\ell_{\infty}(G)\right)$.

Let $\mathcal{F}$ be an ultrafilter on $\mathbb{N}$ which dominates the (natural) order filter. For $h \in \mathbb{N}$, we define

$$
\psi_{h}:=w^{*}-\lim _{n \rightarrow \mathcal{F}} \delta_{y_{(n, h)}} \in \overline{\delta_{G}} w^{*} \subset \operatorname{Ball}\left(\ell_{\infty}(G)^{*}\right)
$$

We now show that the factorizations

$$
f_{h}=\psi_{h} \odot f
$$

and

$$
f_{h}=f \diamond \psi_{h}
$$

hold for all $h \in \mathbb{N}$.

We first show the following results for all $(n, h),(m, k),(l, c) \in \tilde{I}$ with $(l, c) \prec(m, k):$ 


$$
\begin{aligned}
u_{(l, c)}\left(r_{y_{(m, k)}} r_{y_{(n, h)}^{-1}}\left(u_{(n, h)} f_{h}\right)\right) & =u_{(l, c)}\left(u_{(m, k)} r_{y_{(m, k)}} r_{y_{(n, h)}^{-1}}\left(u_{(n, h)} f_{h}\right)\right) \\
& =u_{(l, c)}\left(r_{y_{(m, k)}}\left(r_{y_{(m, k)}^{-1}} u_{(m, k)}\right) r_{y_{(n, h)}^{-1}}\left(u_{(n, h)} f_{h}\right)\right) \\
& =\delta_{(n, h),(m, k)} u_{(l, c)}\left(r_{y_{(m, k)}}\left(r_{y_{(m, k)}^{-1}} u_{(m, k)} f_{k}\right)\right) \\
& =\delta_{(n, h),(m, k)} u_{(l, c)} u_{(m, k)} f_{k} \\
& =\delta_{(n, h),(m, k)} u_{(l, c)} f_{k} .
\end{aligned}
$$

$$
\begin{aligned}
u_{(l, c)}\left(r_{y_{(m, k)}} l_{y_{(n, h)}^{-1}}\left(u_{(n, h)} f_{h}\right)\right) & =u_{(l, c)}\left(u_{(m, k)} r_{y_{(m, k)}} l_{y_{(n, h)}^{-1}}\left(u_{(n, h)} f_{h}\right)\right) \\
& =u_{(l, c)}\left(r_{y_{(m, k)}}\left(r_{y_{(m, k)}^{-1}} u_{(m, k)}\right) l_{y_{(n, h)}^{-1}}\left(u_{(n, h)} f_{h}\right)\right) \\
& =u_{(l, c)}\left(r_{y_{(m, k)}}\left(\chi_{A_{(m, k)} y_{(m, k)}}\right)\left(\chi_{y_{(n, h)} A_{(n, h)}}\right)\left(l_{y_{(n, h)}^{-1}} f_{h}\right)\right) \\
& =\delta_{(n, h),(m, k)} u_{(l, c)}\left(r_{y_{(m, k)}}\left(\chi_{\left\{y_{(m, k)}\right\}}\right)\left(l_{\left.\left.y_{(n, h)}^{-1}, f_{h}\right)\right)}\right)\right. \\
& =\delta_{(n, h),(m, k)} u_{(l, c)} \chi_{\{e\}}\left(r_{y_{(m, k)}}\left(l_{y_{(m, k)}^{-1}} f_{k}\right)\right) \\
& =\delta_{(n, h),(m, k)} u_{(l, c)} \chi_{\{e\}} f_{k} .
\end{aligned}
$$

$$
\begin{aligned}
u_{(l, c)}\left(l_{y_{(m, k)}} l_{y_{(n, h)}^{-1}}\left(u_{(n, h)} f_{h}\right)\right) & =u_{(l, c)}\left(u_{(m, k)} l_{y_{(m, k)}} l_{y_{(n, h)}^{-1}}\left(u_{(n, h)} f_{h}\right)\right) \\
& =u_{(l, c)}\left(l_{y_{(m, k)}}\left(l_{y_{(m, k)}^{-1}} u_{(m, k)}\right) l_{y_{(n, h)}^{-1}}\left(u_{(n, h)} f_{h}\right)\right) \\
& =\delta_{(n, h),(m, k)} u_{(l, c)}\left(l_{y_{(m, k)}}\left(l_{y_{(m, k)}^{-1}} u_{(m, k)} f_{k}\right)\right) \\
& =\delta_{(n, h),(m, k)} u_{(l, c)} u_{(m, k)} f_{k} \\
& =\delta_{(n, h),(m, k)} u_{(l, c)} f_{k} .
\end{aligned}
$$




$$
\begin{aligned}
u_{(l, c)}\left(l_{y_{(m, k)}} r_{y_{(n, h)}^{-1}}\left(u_{(n, h)} f_{h}\right)\right) & =u_{(l, c)}\left(u_{(m, k)} l_{y_{(m, k)}} r_{y_{(n, h)}^{-1}}\left(u_{(n, h)} f_{h}\right)\right) \\
& =u_{(l, c)}\left(l_{y_{(m, k)}}\left(l_{y_{(m, k)}^{-1}} u_{(m, k)}\right) r_{y_{(n, h)}^{-1}}\left(u_{(n, h)} f_{h}\right)\right) \\
& =u_{(l, c)}\left(l_{y_{(m, k)}}\left(\chi_{y_{(m, k)} A_{(m, k)}}\right)\left(\chi_{A_{(n, h)} y_{(n, h)}}\right)\left(r_{y_{(n, h)}^{-1}} f_{h}\right)\right) \\
& =\delta_{(n, h),(m, k)} u_{(l, c)}\left(l_{y_{(m, k)}}\left(\chi_{\left\{y_{(m, k)}\right\}}\right)\left(r_{y_{(n, h)}^{-1}} f_{h}\right)\right) \\
& =\delta_{(n, h),(m, k)} u_{(l, c)} \chi_{\{e\}}\left(l_{y_{(m, k)}}\left(r_{y_{(m, k)}^{-1}} f_{k}\right)\right) \\
& =\delta_{(n, h),(m, k)} u_{(l, c)} \chi_{\{e\}} f_{k} .
\end{aligned}
$$

$$
\begin{aligned}
u_{(l, c)} r_{y_{(m, k)}}\left(\chi_{y_{(n, h)}} g_{h}\right) & =u_{(l, c)}\left(u_{(m, k)} r_{y_{(m, k)}}\left(\chi_{y_{(n, h)}} g_{h}\right)\right) \\
& =u_{(l, c)}\left(r_{y_{(m, k)}}\left(r_{y_{(m, k)}^{-1}} u_{(m, k)}\right)\left(\chi_{y_{(n, h)}} g_{h}\right)\right) \\
& =u_{(l, c)}\left(r_{y_{(m, k)}}\left(\chi_{A_{(m, k)} y_{(m, k)}}\right)\left(\chi_{y_{(n, h)}} g_{h}\right)\right) \\
& =\delta_{(n, h),(m, k)} u_{(l, c)}\left(r_{y_{(m, k)}} \chi_{y_{(m, k)}} g_{k}\right) \\
& =\delta_{(n, h),(m, k)} u_{(l, c)} \chi_{\{e\}} r_{y_{(m, k)}} g_{k} \\
& =\delta_{(n, h),(m, k)} u_{(l, c)} \chi_{\{e\}} g_{k} .
\end{aligned}
$$

$$
\begin{aligned}
u_{(l, c)} l_{y_{(m, k)}}\left(\chi_{y_{(n, h)}} g_{h}\right) & =u_{(l, c)}\left(u_{(m, k)} l_{y_{(m, k)}}\left(\chi_{y_{(n, h)}} g_{h}\right)\right) \\
& =u_{(l, c)}\left(l_{y_{(m, k)}}\left(l_{y_{(m, k)}^{-1}} u_{(m, k)}\right)\left(\chi_{y_{(n, h)}} g_{h}\right)\right) \\
& =u_{(l, c)}\left(l_{y_{(m, k)}}\left(\chi_{y_{(m, k)} A_{(m, k)}}\right)\left(\chi_{y_{(n, h)}} g_{h}\right)\right) \\
& =\delta_{(n, h),(m, k)} u_{(l, c)}\left(l_{y_{(m, k)}} \chi_{y_{(m, k)}} g_{k}\right) \\
& =\delta_{(n, h),(m, k)} u_{(l, c)} \chi_{\{e\}} l_{y_{(m, k)}} g_{k} \\
& =\delta_{(n, h),(m, k)} u_{(l, c)} \chi_{\{e\}} g_{k} .
\end{aligned}
$$


Fix $x \in G$. By the above results we obtain for all $k \in \mathbb{N}$ and $(l, c) \in \tilde{I}$ :

$$
\begin{aligned}
& u_{(l, c)}(x)\left\langle\psi_{k}, l_{x} f\right\rangle \\
= & \lim _{m \rightarrow \mathcal{F}} u_{(l, c)}(x)\left(r_{y_{(m, k)}} f\right)(x) \\
= & \lim _{m \rightarrow \mathcal{F}} \sum_{n \in \mathbb{N}} \sum_{h \in J} u_{(l, c)}(x) r_{y_{(m, k)}}\left[r_{y_{(n, h)}^{-1}}\left(u_{(n, h)} f_{h}\right)+l_{y_{(n, h)}^{-1}}\left(u_{(n, h)} f_{h}\right)-\chi_{y_{(n, h)}} g_{h}\right](x) \\
= & \lim _{m \rightarrow \mathcal{F}} \sum_{n \in \mathbb{N}} \sum_{h \in J}\left[u_{(l, c)}(x) r_{y_{(m, k)}} r_{y_{(n, h)}^{-1}}\left(u_{(n, h)} f_{h}\right)(x)+\right. \\
& \left.\quad+u_{(l, c)}(x) r_{y_{(m, k)}} l_{y_{(n, h)}^{-1}}\left(u_{(n, h)} f_{h}\right)(x)-u_{(l, c)}(x) r_{y_{(m, k)}} \chi_{y_{(n, h)}} g_{h}(x)\right] \\
= & \lim _{m \rightarrow \mathcal{F}} \sum_{n \in \mathbb{N}} \sum_{h \in J}\left[\delta_{(n, h),(m, k)} u_{(l, c)}(x) f_{k}(x)+\delta_{(n, h),(m, k)} u_{(l, c)}(x) \chi_{\{e\}} f_{k}(x)-\right. \\
= & \lim _{m \rightarrow \mathcal{F}} \sum_{n \in \mathbb{N}} \sum_{h \in J} \delta_{(n, h),(m, k),(m, k)} u_{(l, c)}(x) u_{(l, c)}(x)\left[f_{k e\}} g_{k}(x)+\chi_{\{e\}} f_{k}(x)-\chi_{\{(e\}} g_{k}(x)\right] \\
= & \lim _{m \rightarrow \mathcal{F}} \sum_{n \in \mathbb{N}} \sum_{h \in J} \delta_{(n, h),(m, k)} u_{(l, c)}(x) f_{k}(x) \\
= & u_{(l, c)}(x) f_{k}(x) .
\end{aligned}
$$

Since $u_{(l, c)} \longrightarrow 1$ pointwise, we obtain

$$
f_{k}=\psi_{k} \odot f
$$

for all $k \in \mathbb{N}$. 
Similarly,

$$
\begin{aligned}
& u_{(l, c)}(x)\left\langle\psi_{k}, r_{x} f\right\rangle \\
= & \lim _{m \rightarrow \mathcal{F}} u_{(l, c)}(x)\left(l_{y_{(m, k)}} f\right)(x) \\
= & \lim _{m \rightarrow \mathcal{F}} \sum_{n \in \mathbb{N}} \sum_{h \in J} u_{(l, c)}(x) l_{y_{(m, k)}}\left[r_{y_{(n, h)}^{-1}}\left(u_{(n, h)} f_{h}\right)+l_{y_{(n, h)}^{-1}}\left(u_{(n, h)} f_{h}\right)-\chi_{y_{(n, h)}} g_{h}\right](x) \\
= & \lim _{m \rightarrow \mathcal{F}} \sum_{n \in \mathbb{N}} \sum_{h \in J}\left[u_{(l, c)}(x) l_{y_{(m, k)}} r_{y_{(n, h)}^{-1}}\left(u_{(n, h)} f_{h}\right)(x)+\right. \\
= & \lim _{m \rightarrow \mathcal{F}} \sum_{n \in \mathbb{N}} \sum_{h \in J}\left[\delta_{(n, h),(m, k)} u_{(l, c)}(x) \chi_{\{e\}} f_{k}(x)+\delta_{(n, h),(m, k)} u_{(l, c)}(x) f_{k}(x)-\right. \\
= & \lim _{m \rightarrow \mathcal{F}} \sum_{n \in \mathbb{N}} \sum_{h \in J} \delta_{(n, h),(m, k)} u_{(l, c)}(x)\left[\chi_{\{e\}} f_{k}(x)+f_{k}(x)-\chi_{\{(n, h),(m, k)} u_{(l, c)}(x) \chi_{\{e\}} g_{k}(x)\right] \\
= & \lim _{m \rightarrow \mathcal{F}} \sum_{n \in \mathbb{N}} \sum_{h \in J} \delta_{(n, h),(m, k)} u_{(l, c)}(x) f_{k}(x) \\
= & u_{(l, c)}(x) f_{k}(x) \\
& \text { and again as } u_{(l, c)} \longrightarrow \mathbb{1} \text { pointwise, we obtain }
\end{aligned}
$$

$$
f_{k}=f \diamond \psi_{k}
$$

for all $k \in \mathbb{N}$.

Finally we have,

$$
f_{k}=\frac{1}{2}\left(f_{k}+f_{k}\right)=\frac{1}{2}\left(\psi_{k} \odot f+f \diamond \psi_{k}\right)=\psi_{k} \odot_{J} f
$$

which completes the proof.

We now come to our main result.

Theorem 5.2.3 Let $G$ be a locally compact, noncompact abelian group and let $H$ be a countable ICC group. Then $L_{1}(G)$ and $\ell_{1}(H)$ are both Jordan strongly Arens irregular.

Proof Since $G$ is an abelian group then $L_{1}(G)_{J}=L_{1}(G)$ and thus $L_{1}(G)$ is Jordan strongly Arens irregular. By the previous theorem we have that $\ell_{1}(H)^{*}$ enjoys the simultaneous $\ell_{1}(H)^{* *}$-factorization property of level $\aleph_{0}$. Thus by Theorem 5.2 .1 we have that $Z_{t}\left(\ell_{1}(H)_{J}^{* *}\right)=\ell_{1}(H)$. 


\subsection{Jordan topological centre of the convolution algebra of nuclear operators}

From the last section we have found that for $G$ an abelian group or a countable ICC group we have that $\ell_{1}(G)$ is JSAI. Since $\mathcal{N}\left(\ell_{p}(G)\right)=\ell_{1}(G) \oplus\left(M_{\ell_{\infty}(G)}\right)_{\perp}$ we can now determine the Jordan centre for $\mathcal{N}\left(\ell_{p}(G)\right)$ for these two classes of groups.

Theorem 5.3.1 Let $G$ be a countable ICC group or a discrete abelian group. Then $\mathcal{N}\left(\ell_{p}(G)\right)$ is $J S A I$.

Proof Recall that $\mathcal{N}\left(\ell_{p}(G)\right)=\ell_{1}(G) \oplus\left(M_{\ell_{\infty}(G)}\right)_{\perp}$ and $\mathcal{N}\left(\ell_{p}(G)\right)^{* *}=\ell_{1}(G)^{* *} \oplus\left(M_{\ell_{\infty}(G)}\right)^{\perp}$. Let $\Phi=(\rho, \tau) \in Z_{t}\left(\mathcal{N}\left(\ell_{p}(G)\right)_{J}^{* *}\right)$. We first show that $\rho \in \ell_{1}(G)$. So, let $\left(\mu_{\alpha}\right)_{\alpha \in I} \subset \ell_{1}(G)^{* *}$ be a $w^{*}$-convergent net converging to 0 . Then $\left(\mu_{\alpha}, 0\right)$ is a net in $\ell_{1}(G)^{* *} \oplus\left(M_{\ell_{\infty}(G)}\right)^{\perp}$ converging $w^{*}$ to 0. As $(\rho, \tau) \in Z_{t}\left(\mathcal{N}\left(\ell_{p}(G)\right)_{J}^{* *}\right)$ then $(\rho, \tau) \odot_{J}\left(\mu_{\alpha}, 0\right)$ converges $w^{*}$ to 0 . Since $(\rho, \tau) \odot_{J}\left(\mu_{\alpha}, 0\right)=\left(\rho \odot_{J} \mu_{\alpha}, \tau \odot_{J} \mu_{\alpha}\right)$ we have that $\rho \odot_{J} \mu_{\alpha}$ converges $w^{*}$ to 0 which gives $\rho \in Z_{t}\left(\ell_{1}(G)_{J}^{* *}\right)=\ell_{1}(G)$. Thus $(\rho, \tau) \in \ell_{1}(G) \oplus\left(M_{\ell_{\infty}(G)}\right)^{\perp}$. By Proposition 5.1 .2 we get that $(\rho, \tau) \in \ell_{1}(G) \oplus\left(M_{\ell_{\infty}(G)}\right)_{\perp}=\mathcal{N}\left(\ell_{p}(G)\right)$. 


\subsection{Conclusion and main results}

As we stated in the introduction, there were three main objectives for our research. These objectives were:

i) to determine the second topological centre of $\left(\mathcal{N}\left(\ell_{p}(G)\right), *\right)$ which demonstrates concretely that $\left(\mathcal{N}\left(\ell_{p}(G)\right), *\right)$ is not right strongly Arens irregular;

ii)to construct a Banach algebra that is strongly Arens irregular that admits a subalgebra that is Arens regular (non-trivially);

iii)to investigate the notion of the Jordan topological centre for $\ell_{1}(G)$ and $\left(\mathcal{N}\left(\ell_{p}(G)\right), *\right)$.

We have successfully shown all three parts except for part (iii) where we have only shown this when $G$ is a discrete abelian group or a countable ICC group. Our technique used to determine (iii), a simultaneous factorization result, is also of interest on its own. This result is a strengthening of the factorization result developed by Neufang in [18]. So far, Neufang's factorization result is the most powerful tool in determining the topological centres of a Banach algebra, and the simultaneous factorization result is an analogous tool for that of Jordan-Banach algebras.

In the investigation of the Jordan topological centre of $\left(\mathcal{N}\left(\ell_{p}(G)\right), *\right)$ it was natural to look at the Jordan centre of $\ell_{1}(G)$. This has inspired some questions that are still unanswered. These questions are:

1) Is $L_{1}(G)$ Jordan strongly Arens irregular for every locally compact group $G$ ?

2) If $G$ is not isomorphic to a locally compact abelian group or a countable ICC group, can $L_{1}(G)^{*}$ still have the simultaneous $L_{1}(G)^{* *}$-factorization property of level $\kappa(G)$, the compact covering number of $G$ ? In particular, when $G$ is the product of a locally compact abelian group and a countable ICC group.

3) Does there exist a Banach algebra where the Jordan centre is strictly bigger than the intersection of the first and the second centre? (Note: this question is trivially yes if we relax the condition of associativity of the original algebra since any Lie algebra is Jordan Arens regular). 


\section{Bibliography}

[1] O.Y. Aristov. Banach Algebras and Their Applications. Contemporary Mathematics 363. Sixteenth International Conference on Banach Algebras, University of Alberta 2003. pp.15-37.

[2] B. Bollobás. Linear Analysis. Cambridge University Press 1999.

[3] D.L. Cohn. Measure Theory. Boston, Birkhäuser 1980.

[4] G. Dales. Introduction to Banach algebras, operators, and harmonic analysis. New York, Cambridge University Press 2003.

[5] G. Dales and A.T. Lau,. The Second Duals of Beurling Algebras. Memoirs of the American Mathematical Society 177 (2005) no.836, pp.40-41.

[6] E.G. Effros and Z.Ruan. Operator Spaces. New York, Oxford University Press Inc. 2000 .

[7] M. Fabian et al. Functional Analysis and Infinite-Dimensional Geometry. Springer-Verlag New York, Inc. 2001.

[8] G.B. Folland. A course in Abstract Harmonic Analysis. Boca Raton, CRC Press. 1995.

[9] F. Ghahramani, J.P. McClure, and M. Meng. On Asymmetry of topological Centers of the Second Duals of Banach Algebras. Proceedings of the American Mathematical Society 126 (1998), no.6, pp.1765-1768

[10] M.E. Gordji and M. Filali. Arens regularity of module actions. Preprint. 
[11] E. Hewitt and K.A. Ross. Abstract Harmonic Analysis. Volume 1. Berin, Springer 1970.

[12] Z. Hu and M. Neufang. Decomposability of von Neumann Algebras and the Mazur Property of Higher Level. Canadian Journal of Mathematics 58 (2006) no.4, pp.768-795.

[13] A.T. Lau and V. Losert. On the second conjugate algebra of $L_{1}(G)$ of a locally compact group. Journal of the London Mathematical Society. Second Series 37 (1988) no.3,

pp 464-470.

[14] R.E. Megginson. An Introduction to Banach Space Theory. Springer-Verlag New York, Inc. 1998.

[15] M. Neufang. On one-sided strong Arens irregularity. Preprint.

[16] M. Neufang. Isometric Representation of Convolution Algebras as Completely Bounded Module Homomorphisms and a Characterization of the Measure Algebra. Preprint.

[17] M. Neufang. On a conjecture by Ghahramani-Lau and related problems concerning topological centres. Journal of Functional Analysis 224 (2005) no.1, pp.217229 .

[18] M. Neufang. Abstrakte Harmonische Analyse und Modulhomomorphismen über von Neumann-Algebren. Ph.D. Thesis. Univerität des Sarlandes (2000).

[19] M. Neufang. A unified approach to the topological centre problem for certain Banach algebras arising in abstract harmonic analysis. Archiv der Mathematik (Basel) 82 (2004) no.2, pp.164-171.

[20] M. Neufang. Solution to a conjecture by Hofmeier-Wittstock. Journal of Functional Analysis 217 (2004) no.1, pp.171-180. 
[21] T.W. Palmer. Banach Algebras and the General Theory of *-Algebras. New York, Cambridge University Press 1994.

[22] A.Y. Pirkovskii. Biprojectivity and Biflatness for Convolution Algebras of Nuclear Operators. Canadian Mathematical Bulletin 47 (2003) no.3, pp.445-455.

[23] S. Willard. Introduction to general topology. Addison-Wesley Publishing Company 1970.

[24] R.A. Ryan. Introduction to Tensor Products of Banach Spaces. Springer-Verlag London Limited 2002. 